\title{
Hepcidin Promoted Ferroptosis through Iron Metabolism which Is Associated with DMT1 Signaling Activation in Early Brain Injury following Subarachnoid Hemorrhage
}

\author{
Hongxia Zhang, ${ }^{1}$ Robert Ostrowski, ${ }^{2}$ Dengzhi Jiang, ${ }^{1}$ Qing Zhao, ${ }^{1}$ Yidan Liang, \\ Xudong Che, ${ }^{1}$ Jun Zhao, ${ }^{1}$ Xiang Xiang, ${ }^{1}$ Wang Qin, ${ }^{1}$ and Zhaohui He ${ }^{1}$ \\ ${ }^{1}$ Department of Neurosurgery, the 1st Affiliated Hospital of Chongqing Medical University, Chong Qing, China \\ ${ }^{2}$ Department of Experimental and Clinical Neuropathology, Mossakowski Medical Research Centre Polish Academy of Sciences, \\ Warsaw, Poland \\ Correspondence should be addressed to Zhaohui He; geno_he@163.com
}

Received 12 October 2021; Revised 26 November 2021; Accepted 2 December 2021; Published 27 December 2021

Academic Editor: Kamil Duris

Copyright (c) 2021 Hongxia Zhang et al. This is an open access article distributed under the Creative Commons Attribution License, which permits unrestricted use, distribution, and reproduction in any medium, provided the original work is properly cited.

\begin{abstract}
Iron metabolism disturbances play an important role in early brain injury (EBI) after subarachnoid hemorrhage (SAH), and hepcidin largely influences iron metabolism. Importantly, iron metabolism may be associated with ferroptosis, recently a nonapoptotic iron-dependent form of cell death that may have a great impact on brain injury after SAH. We investigated hepcidin on iron metabolism and ferroptosis involving divalent metal transporter 1 (DMT1), and ferroportin-1 (FPN1) in a rat model of SAH. Male Sprague-Dawley rats were subjected to the endovascular perforation to induce SAH, and treated with heparin (inhibitor of hepcidin), or oncostatin M (OSM, inducer of hepcidin), or ebselen (inhibitor of DMT1) by intracerebroventricular injections. Hepcidin, DMT1, FPN1 and glutathione peroxidase 4 (GPX4), were detected by western blot and immunofluorescence. Iron metabolism was detected through Perl's iron staining and iron content assay. Ferroptosis, the ROS production, lipid peroxidation (LPO) was evaluated by monitoring methane dicarboxylic aldehyde (MDA), glutathione (GSH), glutathione peroxidase 4 (GPX4) activity, and transmission electron microscopy. Neurological deficit scores, Evans blue staining and brain water content were also determined to detect EBI $72 \mathrm{~h}$ after SAH. Our results showed that inhibition of DMT1 by ebselen could suppress iron accumulation and lipid peroxidation, and thereby alleviate ferroptosis and EBI in SAH rats. Heparin downregulated the expression of hepcidin and DMT1, increased FPN1, and exerted protective effects that were equivalent to those of ebselen on ferroptosis and EBI. In addition, OSM increased the expression of hepcidin and DMT1, decreased FPN1, and aggravated ferroptosis and EBI, while the effect on ferroptosis was reversed by ebselen. Therefore, the study revealed that hepcidin could regulate iron metabolism and contribute to ferroptosis via DMT1 signaling activation in rats with EBI after SAH.
\end{abstract}

\section{Introduction}

Subarachnoid hemorrhage (SAH) is a devastating condition. Increasing evidence indicates that early brain injury (EBI), a recently proposed concept referring to a direct damage to the whole brain within $72 \mathrm{~h}$ after SAH, is the most crucial etiological factor of poor clinical prognosis amongst $\mathrm{SAH}$ cases $[1,2]$. However, the mechanism of EBI in SAH is still poorly understood. Our previous research focused on the iron metabolism in EBI after $\mathrm{SAH}$, it reported that iron metabolism may induce ferroptosis, a new form of cell death. Ferroptosis mainly caused by iron overload and the accumulation of lipid peroxide [3]. It has reported ferroptosis occurs after SAH, and reduction of lipid peroxidation could alleviate ferroptosis in early brain injury after $\mathrm{SAH}$ [4]. Whether the disorder of iron metabolism might lead to ferroptosis after SAH has not reported, and the pathway also not. Studies revealed that hepcidin had a potent 
influence on iron metabolism [5], moreover, our group has found that the expression hepcidin increased in EBI after $\mathrm{SAH}[6]$.

Noteworthy, a 2012 study by Dixon et al. indicated that ferroptosis, a nonapoptotic form of cell death, is dependent on intracellular iron-induced accumulation of lipid peroxidation (LPO) products due to reactive oxygen species (ROS), a decreased glutathione (GSH) content, and is manifested by the reduced size of mitochondria [3]. Emerging evidence suggests that iron accumulation and lipid peroxidation, accompanied by reductions in GSH and glutathione peroxidase 4 (GPX4), the main regulator of ferroptosis, which levels can be found in various neurological diseases [7-10]. What's more, the cellular iron-regulatory system is complex and involves various proteins that collectively regulate import, neutralization, storage and export of iron, mainly denoted by the transferrin receptor 1 , divalent metal transporter 1 (DMT1), ferritin and ferroportin-1 [11]. Among them, DMT1 protein facilitates iron uptake at the apical cell membrane, and transports iron across the endosomal membrane in almost all cell types that take up iron via transferrin/transferrin receptor 1 pathway [12]. While, ferroportin-1 (FPN1) is one of the extracellular iron transporters, which exports iron across the basolateral membrane. Previous studies indicated that hepcidin, an iron regulatory hormone, could promote iron accumulation through inhibiting FPN1 and ceruloplasmin (CP) and activation DMT1 in the rat cerebral cortex and hippocampus [13]. What's more, ferroptosis was induced by iron deposition, studies found the contrary expression of DMT1 and GPX4 at the same time. Zhang et al. found that lacking Pkd1 exhibit extensive metabolic abnormalities, which include increased expression of iron importers DMT1, which in turn result in high iron levels, low GSH and GPX4 activity, increased lipid peroxidation, and propensity to ferroptosis in autosomal dominant polycystic kidney disease mouse models [14] Zeng et al. showed that a high expression DMT1, at the same time, the low expression of ferroptosis key factor GPX4 was detected in studying the benefits of iron chelators in the treatment of Parkinson's disease [15]. Therefore, we hypothesized that hepcidin could regulate iron metabolism via DMT1 signaling activation and FPN1 inhibition, which promote iron accumulation and induce ferroptosis in EBI after SAH (Figure 1).

Our group have verified that the role of hepcidin might induce apoptosis after EBI in SAH [6], in this study, we further studied the hypothesis of the hepcidin and its pathway on iron metabolism in ferroptosis in SAH. To test the hypothesis, we initially investigated the expression of hepcidin, DMT1, FPN1, and GPX4 in EBI after SAH. We subsequently administered an inhibitor of DMT1, ebselen (also called PZ51), ebselen was shown to significantly decrease iron concentrations [16]. Ebselen treatment is associated with reduced tissue iron in a model of iron overload [17], suggesting its potential inhibition of iron uptake. What's more, it has been demonstrated that ebselen is an inhibitor of DMT1 transport [18, 19]. Ebselen in vivo and investigated the iron metabolism associated with iron content and Perl's iron staining. Moreover, heparin, an inhibitor of hepcidin, was used to investigate the functions of hepcidin in iron metabolism and ferroptosis in EBI induced by SAH. Heparin is a glycosaminoglycan analog to HSPGs, studies showed that heparin is a strong inhibitor of hepcidin expression in hepatic cell lines, in mice, and possibly also in patients with high hepcidin levels, lines also reported evidence that heparin has a strong anti-hepcidin activity in vitro and in vivo [20]. What's more, studies showed that unfractionated heparin strongly inhibits hepcidin expression in HepG2 cells and the unfractionated heparin (UFH, 12$15 \mathrm{kDa}$ ) had an effect approximately 10 -fold more potent than LMWH $(4.5 \mathrm{kDa})$. To avoid its anticoagulant activity, the glycol-split non-anticoagulant heparin was introduced into research (heparin is abbreviated form of glycol-split heparin in the following text) [21]. Oncostatin M (OSM), an inducer of hepcidin, OSM is an inflammatory cytokine member of the IL-6-related cytokine family, OSM expression was demonstrated in T-cells, monocytes, neutrophils, and also in the testis, brain, and kidne1 [22]. A possible physiologically relevant role for OSM induced hepcidin gene expression could be during fetal liver development, OSM might be more important for hepcidin induction in physio-pathological conditions than IL-6 and that OSM may play an important role in iron dysregulation observed in inflammatory condition $[23,24]$. OSM was introduced to investigate the functions of hepcidin in iron metabolism and ferroptosis in EBI induced by SAH. Finally, the mechanism involving DMT1 was also investigated using both ebselen and OSM interventions. Ultimately, our findings reveal that hepcidin and its related signaling pathways after SAH may provide a molecular target for clinical treatment.

\section{Materials and Methods}

2.1. Animals and Grouping. Male Sprague-Dawley (SD) rats were introduced into research, for the present SAH model a total of 383 rats, weighing 250-300 g, were purchased from the Animal Center of Chongqing Medical University. The rats had free access to water and food, and were housed in plastic cages with a $12 \mathrm{~h}$ light/dark cycle, which was in accordance with the National Institute of Health Guide for the Care and Use of Laboratory Animals (NIH Publications No. 80-23) revised 1996. Before surgery, the rats were fasted for $12 \mathrm{~h}$ and deprived of water for $8 \mathrm{~h}$. The animal care and all experimental protocols adhered to the guidelines of and were approved by the Institutional Medical Experimental Animal Care Committee of Chongqing Medical University.

The adult male SD rats assigned to SAH model procedures were randomly divided into several groups. The rats assigned to $\mathrm{SAH}$ model procedures were randomly divided into the groups, first to determine the expression of hepcidin, DMT1, FPN1, and GPX4, the main regulator of ferroptosis, and to subsequently select the most suitable timing for drug injections. Second, adult male SD rats were randomly divided into the groups to determine the significant preoperative doses of ebselen, heparin and OSM in terms of their effects on hepcidin, DMT1, FPN1, and GPX4 for further study. Lastly, male SD rats were randomly divided into the groups to determine the effects of hepcidin and DMT1 on 


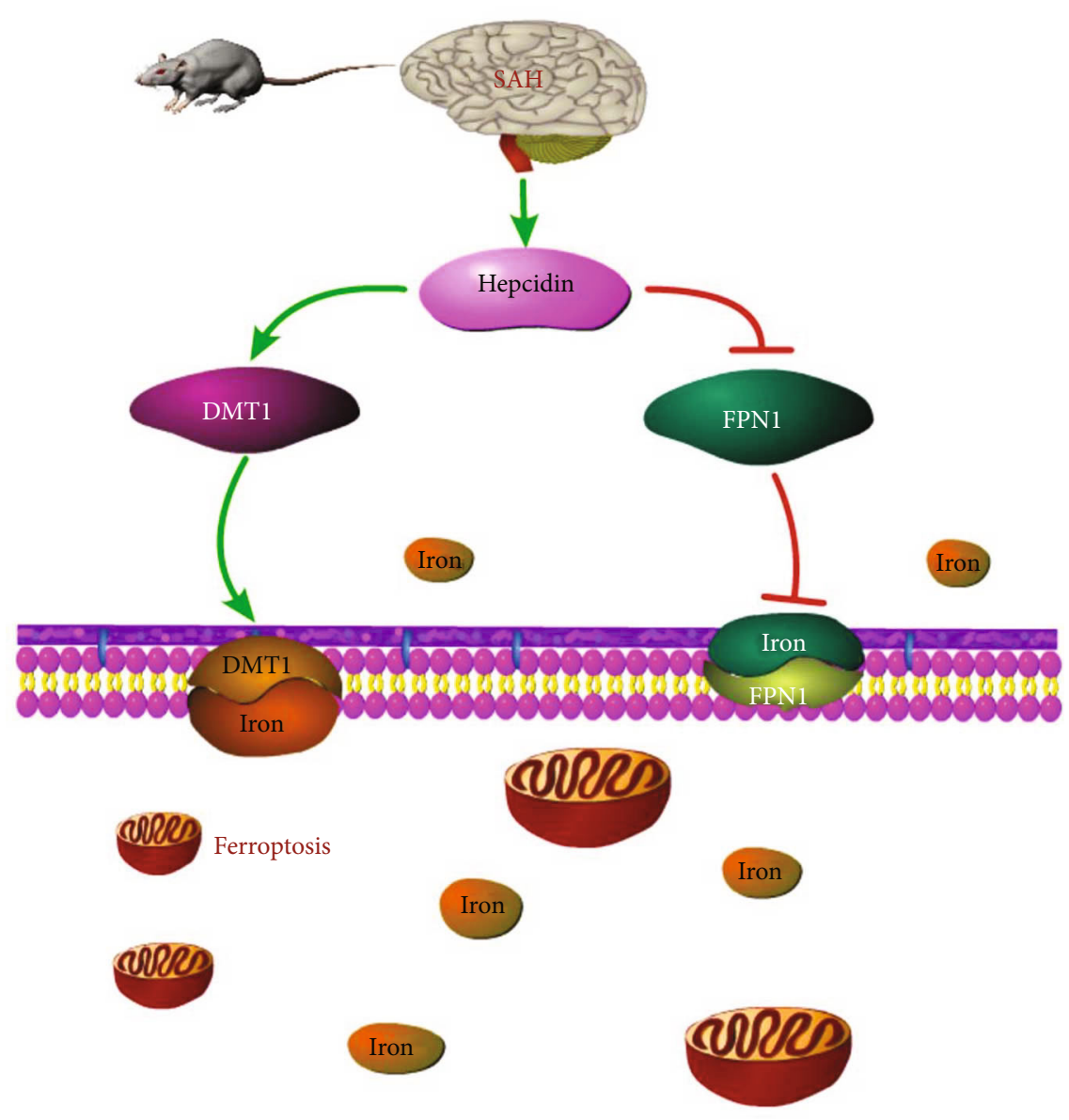

FIGURE 1: hepcidin might have an effect on iron metabolism and ferroptosis, via DMT1 activation, in EBI after SAH.

iron metabolism, ferroptosis, and EBI, by using heparin, ebselen and OSM as the experimental interventions.

2.2. Rat Model of SAH. The endovascular perforation model is considered be in similar with the pathophysiological changes observed with a clinical ruptured intracranial aneurysm, which is the most suitable model of SAH [25]. Takashi Sugawara et.al outlined a simple and objective novel SAH grading system by examining endovascular perforation of subarachnoid blood clots in the basal castle and evaluating its correlation with neurological status [26]. They found that the new SAH severity scale was linearly associated with neurological dysfunction, which is a more intuitive and effective evaluation of the SAH model. SAH animal models were established through endovascular perforation as previously described [27]. The rats were anesthetized by intraperitoneal injection of pentobarbital sodium $(50 \mathrm{mg} / \mathrm{kg})$, and a sharpened 4-0 monofilament nylon suture was inserted into the right internal artery from the stump of the external carotid artery to puncture the bifurcation of the anterior and middle cerebral arteries. The sham-operated group underwent the same procedure as the SAH group, but without puncturing the vessel wall. As mentioned earlier, at the time of euthanasia, the severity of SAH was assessed by investigators who were unaware of the group ID, and rats with scores $<9$ were excluded from the study.

\subsection{Treatments}

2.3.1. Treatment 1. Western blot was performed to determine the expression profiles of hepcidin, DMT1, FPN1, and GPX4 at $6,12,24,48$, and $72 \mathrm{~h}$ after SAH $(n=6$, each group, Figure 2).

2.3.2. Treatment 2. Ebselen (DMT1 inhibitor) at doses of 1,2, and $4 \mathrm{mg} / \mathrm{kg}$ was injected intracerebroventricularly $24 \mathrm{~h}$ before $\mathrm{SAH}$ constructed, the vehicle groups received $10 \%$ absolute alcohol (Abs) injection ( $n=6$, each group, Figure 2$)$. Heparin (hepcidin inhibitor) at doses of $1,2.5$, and $5 \mathrm{mg} / \mathrm{kg}$ was injected intracerebroventricularly $24 \mathrm{~h}$ before SAH constructed, the vehicle groups received normal saline (NS) injection ( $n=6$, each group, Figure 2). OSM (hepcidin inducer) at doses of $100 \mathrm{ng}, 1,5$, and $10 \mu \mathrm{g}$ was injected intracerebroventricularly, the vehicle groups received a phosphate-buffered saline (PBS) injection ( $n=6$, each group, Figure 2$)$. Western blot to evaluate the expression of hepcidin, DMT1, FPN1, and GPX4 to get the proper concentration of ebselen, heparin and OSM. The proper concentration of ebselen, heparin and OSM were chosen to evaluate ferroptosis and EBI after SAH $(n=6$, each group, Figure 2).

2.3.3. Treatment 3. The proper concentration of OSM and ebselen were injected intracerebroventricularly $24 \mathrm{~h}$ before 


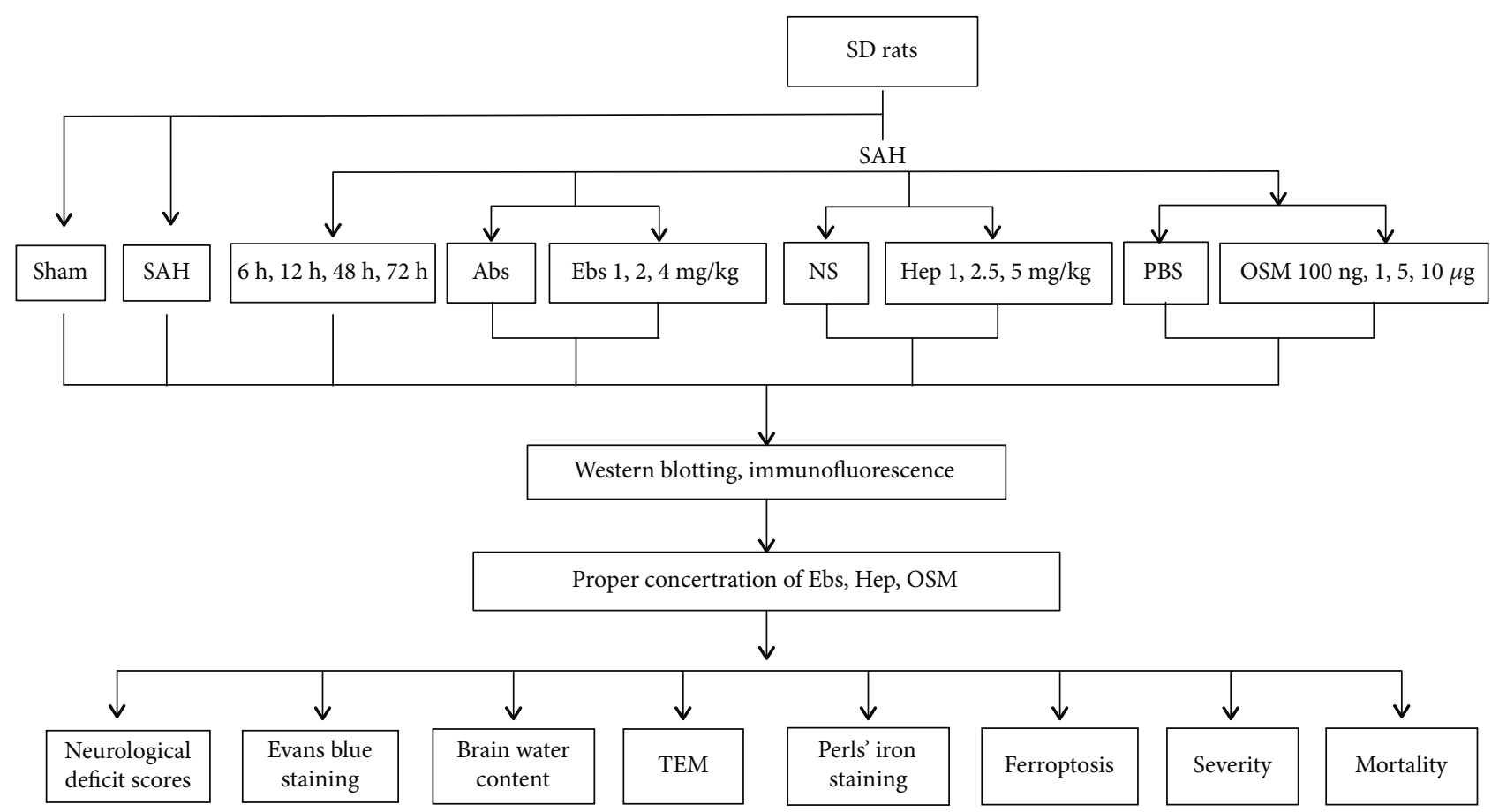

FIGURE 2: The proper concentration of ebselen, heparin and OSM were chosen to evaluate the ferroptosis and EBI after SAH.

SAH constructed. The vehicle groups received equal volumes of PBS and Abs ( $n=6$, each group, Figure 2).

2.4. Immunofluorescence. Rats were sacrificed $24 \mathrm{~h}$ after perforation for immunofluorescence staining using the methods described in our previous study. Ten-micrometer sections of brains from rats with SAH were incubated with the following primary antibodies overnight at $4^{\circ} \mathrm{C}$ : antihepcidin (1:100, Abcam, ab30760), anti-DMT1 (1:300; Bioss, bs-3577R), and anti-NeuN (1:100, Millipore, MAB377X). After washing in PBST, sections were incubated with the following fluorescent secondary antibodies at $37^{\circ} \mathrm{C}$ for 1.5 h: Alexa Fluor 555 (1:50, Beyotime, A0453) and Alexa Fluor 488 (1:50, Protech, SA00006-1). Fluorescence microscopy (Nikon A1+R, Japan) was used to detect the protein locations in the neurons.

2.5. Western Blotting. Western blotting was performed $24 \mathrm{~h}$ after SAH. Equal amounts of total protein $(50 \mu \mathrm{g})$ were loaded onto a sodium dodecyl sulfate polyacrylamide gel, electrophoresed, and transferred to a polyvinylidene fluoride (PVDF) membrane. The membranes were incubated at $37^{\circ} \mathrm{C}$ with $5 \%$ nonfat milk in Tris-buffered saline that contained $0.1 \%$ Tween 20 (TBST) for $2 \mathrm{~h}$, followed by incubation with primary antibodies overnight at $4^{\circ} \mathrm{C}$. The following primary antibodies were used: anti-hepcidin (1:100, Abcam, ab30760), anti-DMT1 (1:300, Bioss, bs-3577R), anti-FPN1 (1:1000, Protech, 26601-1-AP), and anti-GPX4 (1:1000, Protech, 14432-1-AP). The membranes were incubated with the goat anti-rabbit HRP-conjugated secondary antibody $(1: 2000$, BosterBio, BA1054) after washing in TBST. The membranes were visualized and analyzed using
Fusion FX7 Spectra chemiluminescence imaging system (Vilber, France).

2.6. Perl's Iron Staining. Samples were collected $24 \mathrm{~h}$ after SAH. The Perl's iron staining was performed as described previously with some modifications [28]. Paraffin slices 10 microns thick were taken from the brains with SAH rats and incubated with xylene I and xylene II for $15 \mathrm{~min}$, followed by hydration with 100\%, 95\%, 85\%, 80\%, 75\% and $70 \%$ alcohol solutions for $1 \mathrm{~min}$. Incubated with Perl's iron staining $(80 \mathrm{ml} \mathrm{20 \%}$ hydrochloric acid and $80 \mathrm{ml} 10 \%$ potassium ferrocyanide mixed for $5 \mathrm{~min}$ ) for $20-30 \mathrm{~min}$ and washed with distilled water 3 times. After $1 \mathrm{~min}$ of $\mathrm{H} \& \mathrm{E}$ staining, sections were rapidly dehydrated for $5 \mathrm{~s}$ at $80 \%, 85 \%, 90 \%$, and $100 \%$ alcohol concentrations. Finally, the sections were cleaned with xylene and sealed with resin. The stained sections were examined microscopically and multiple fields were evaluated. According to the manufacturer's instructions, Perl's iron staining was introduced into the study to obtain staining with greater detail.

2.7. Iron Content. Samples were collected $24 \mathrm{~h}$ after SAH and homogenized with physiological saline at a ratio of $1: 9$. The iron content was measured using a rat iron assay kit (Nanjing Jian Cheng, A039-2) according to the manufacturer's instructions.

2.8. MDA. Samples were collected $24 \mathrm{~h}$ after SAH and LPO was measured based on the MDA content using an MDA assay kit (Nanjing Jian Cheng, A0003-1) according to the manufacturer's instructions. 
2.9. GSH. Samples were collected $24 \mathrm{~h}$ after SAH and GSH was measured using a GSH assay kit (Nanjing Jian Cheng, A006-2) according to the manufacturer's instructions.

2.10. GPX4 Activity. Samples were collected $24 \mathrm{~h}$ after $\mathrm{SAH}$ and GPX4 was measured using a rat GPX4 assay kit (Nanjing Jian Cheng, A039-2) according to the manufacturer's instructions.

2.11. Transmission Electron Microscopy (TEM). The brains of the SAH rats were stored $24 \mathrm{~h}$ after perfusion with $4 \%$ glutaraldehyde solution, and the cortex of each perfused brain was sliced to a $1 * 1 * 1 \mathrm{~mm}$ size to obtain slices for TEM. Prior to TEM, these slices were subjected to fixing, dehydrating, embedding, curing, thin-slice machine slicing at 50$60 \mathrm{~nm}$, and double staining with 3.3\% uranium-citric acid.

2.12. Neurological Deficit Scores. The scoring method was introduced to evaluate neurological deficit [26], the following six separate tests were scored $24 \mathrm{~h}$ and $72 \mathrm{~h}$ after operation to evaluate the prognosis of SAH rats: independent movement, limb movement symmetry, climbing movement, forepaw extension, response to touch stimulation and beard response. The neurological deficit scores were evaluated by an observer who did not know the group ID and treatment, and Dengzhi Jiang and Qing Zhao scored. The lower the scores, the more serious the neurological impairment.

2.13. Evans Blue Staining. The permeability of blood brain barrier was evaluated by Evans blue staining $72 \mathrm{~h}$ after $\mathrm{SAH}$, as described previously by $\mathrm{He}$ et al. [27] with minor modifications. The rats were anesthetized and directly injected with $2 \%$ Evans blue solution ( $8 \mathrm{ml} / \mathrm{kg}$, Beyotime) through femoral vein. Evans blue solution was allowed to circulate for 3 hours before transcardiac perfusion. After transcardiac perfusion with PBS ( $\mathrm{pH} 7.4)$, the rats were euthanized and the left and right cerebral hemispheres were stored at $-80^{\circ} \mathrm{C}$ before use. Brain tissue was homogenized in 99\% dimethylformamide, and the samples were cultured in $50^{\circ} \mathrm{C}$ water bath for 48 hours. After centrifugation for 30 minutes $\left(12000 \mathrm{~g}\right.$ at $\left.4^{\circ} \mathrm{C}\right)$, the supernatant was collected and the absorbance of the sample was measured at $620 \mathrm{~nm}$ using a spectrophotometer.

2.14. Analysis of Brain Water Content. As mentioned in previously reported [27], the brain water content of rats was detected at $72 \mathrm{~h}$ after SAH induction. Anesthetized the animals and immediately removed the brain. The brain was divided into four parts: the right hemisphere, the left hemisphere, the cerebellum and the brain stem. Weighed each part on an electronic analytical balance to obtain a wet weight (a) and then dried at $100^{\circ} \mathrm{C}$ for $24 \mathrm{~h}$ to obtain the dry weight (b)). The brain water content (c) was calculated by the following formula: $c=(a-b) / a$.

2.15. Statistical Analysis. The data are expressed as the Means \pm SEM. Statistical differences among the groups were analyzed using one-way ANOVA followed by Tukey's post hoc test. Mortality was evaluated using Fisher's exact test. A $p$ value of $<0.05$ was considered statistically significant.
All statistical analyses were performed using GraphPad Prism 5 for Windows (GraphPad software, San Diego, California).

\section{Results}

3.1. Hepcidin and DMT1 Were Upregulated following the Time Course in EBI after SAH. Double immunofluorescence staining revealed that hepcidin, DMT1, and GPX4 were mainly present in the cytoplasm of cortical neurons after SAH $(n=6$, Figure 3(a)). Western blot analysis was performed to determine the expression profiles of hepcidin, DMT1, FPN1, and GPX4. at 6, 12, 24, 48, and $72 \mathrm{~h}$ after SAH (Figure 3(b)). The Western blot results indicated that the hepcidin and DMT1 levels increased with time, and in particular hepcidin was significantly increased from $24 \mathrm{~h}$ to $72 \mathrm{~h}(\mathrm{p}<0.05)(n=6$, Figure $3(\mathrm{c}))$. DMT1 was increased at 6 and $12 \mathrm{~h}$, decreased at $24 \mathrm{~h}$, and significantly increased from $48 \mathrm{~h}$ to $72 \mathrm{~h}(\mathrm{p}<0.05)(n=6$, Figure $3(\mathrm{~d}))$. In contrast, FPN1 was decreased at $6 \mathrm{~h}$, and significantly decreased at $72 \mathrm{~h}(\mathrm{p}<0.05)(n=6$, Figure $3(\mathrm{e}))$. GPX4 gradually decreased during the first $72 \mathrm{~h}$, significantly decreased from $24 \mathrm{~h}$ to $72 \mathrm{~h}$ $(\mathrm{p}<0.05)(n=6$, Figure 3(f)), compared with the sham group.

3.2. DMT1 Inhibition Influenced Iron Metabolism, Resulting in Diminishing Iron Content and Cellular Distribution of Iron Deposits, and Protected against Ferroptosis in EBI following SAH. A suppressant of DMT1, ebselen, was intracerebroventricularly injected at three doses $(1,2$, and $4 \mathrm{mg}$ / $\mathrm{kg}) 24 \mathrm{~h}$ before SAH. The expression of DMT1 was significantly decreased with $4 \mathrm{mg} / \mathrm{kg}$ ebselen $(\mathrm{p}<0.05)$, whereas 1 and $2 \mathrm{mg} / \mathrm{kg}$ ebselen had no effect on DMT1 $(\mathrm{p}>0.05)(n=6$, Figure 3(h)), compared with the Abs+SAH. GPX4 was significantly increased with $4 \mathrm{mg} / \mathrm{kg}$ ebselen $(\mathrm{p}<0.05)$, whereas 1 and $2 \mathrm{mg} / \mathrm{kg}$ ebselen had no influence on GPX4 $(\mathrm{p}>0.05)(n=6$, Figure 3(i)), compared with the Abs+SAH. Ebselen $(4 \mathrm{mg} / \mathrm{kg})$ had a significant effect on DMT1 and GPX4.

Based on the Western blot results, the appropriate dose of ebselen $(4 \mathrm{mg} / \mathrm{kg})$ was administered to examine the role of DMT1 signaling in EBI, iron metabolism, and ferroptosis. Ebselen at a dose of $4 \mathrm{mg} / \mathrm{kg}$ could influence iron metabolism, resulting in decreases in the Perl's iron staining and iron content (Figures 3(j), 3(k)). Ferroptosis was evaluated basing on LPO production of MDA, and GSH content and GPX4 activity were decreased $(\mathrm{p}<0.05)(n=6$, Figures $3(\mathrm{l})$, $3(\mathrm{~m}), 3(\mathrm{n}))$, compared with the Abs+SAH groups. Additionally, $4 \mathrm{mg} / \mathrm{kg}$ ebselen decreased the size of mitochondria of ferroptosis after $\mathrm{SAH}$, and it protected against ferroptosis $(n=6$, Figure $4(\mathrm{i}))$. The results indicated that $4 \mathrm{mg} / \mathrm{kg}$ ebselen improved the neurological deficit, ameliorated the brain edema, and decreased the blood-brain barrier permeability, thus mitigating EBI ( $n=6$, Figures 5(c), 5(d), 5(e)).

3.3. Hepcidin Suppression Decreased the Iron Content and Perl's Iron Staining and Conferred Protection against Ferroptosis and EBI after SAH by Inhibiting DMT1. A hepcidin antagonist, heparin, was administered (1, 2.5, and $5 \mathrm{mg} / \mathrm{kg}$ ) via intracerebroventricular injection $24 \mathrm{~h}$ before $\mathrm{SAH}$ induction. The Western blot results indicated that 

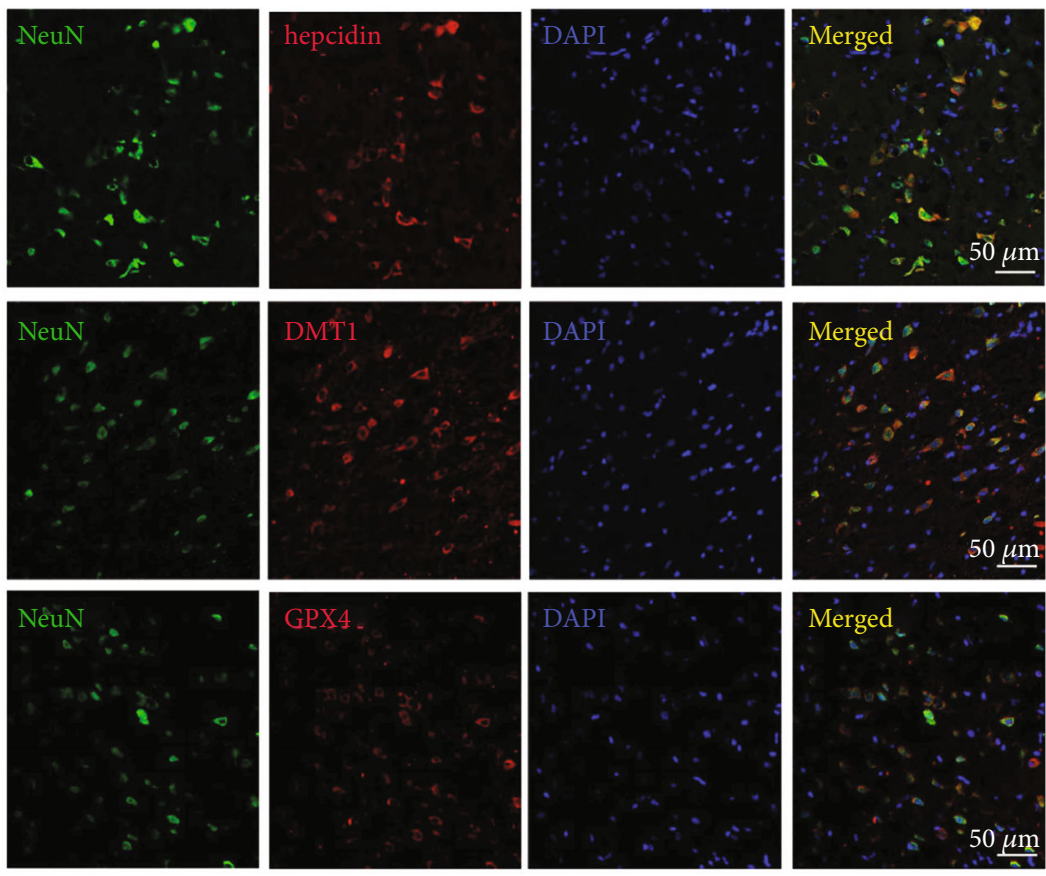

(a)

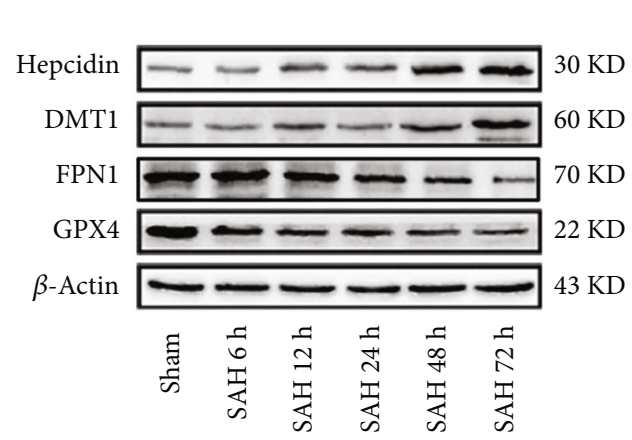

(b)

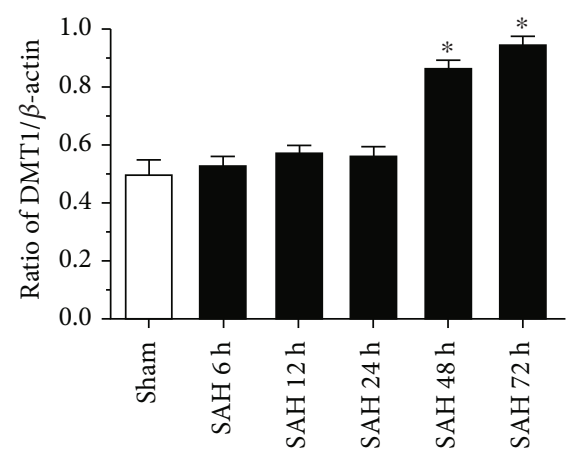

(d)

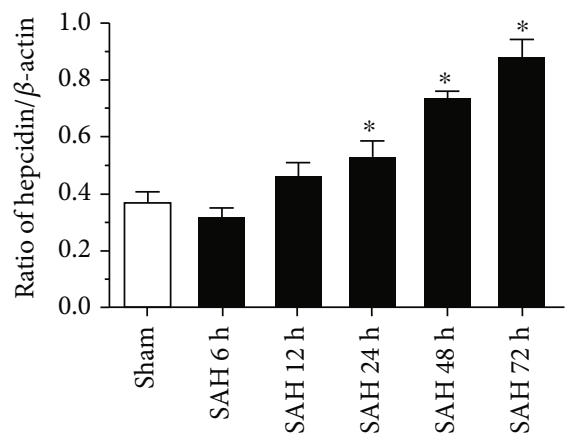

(c)

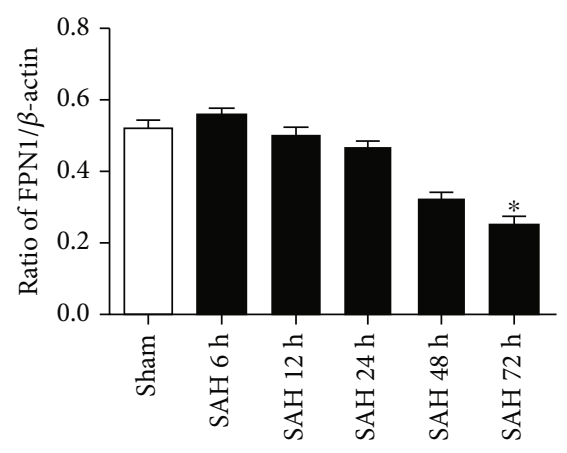

(e)

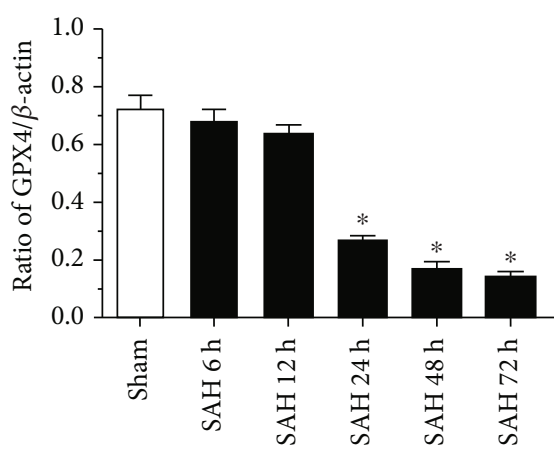

(f)

Figure 3: Continued. 


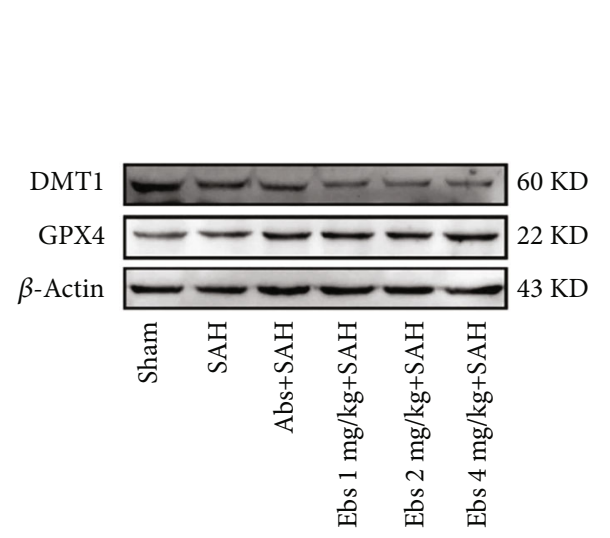

(g)

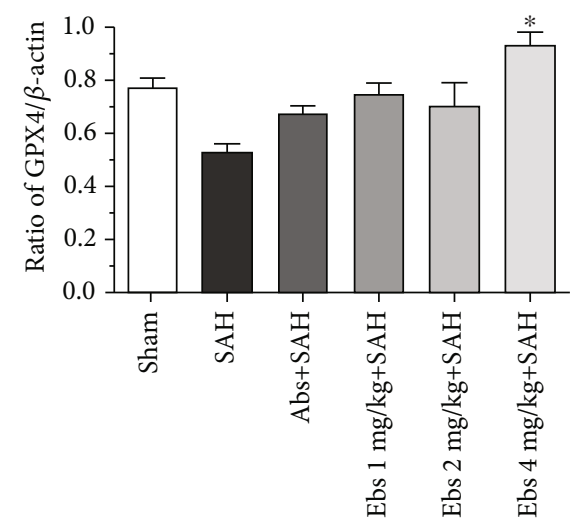

(i)

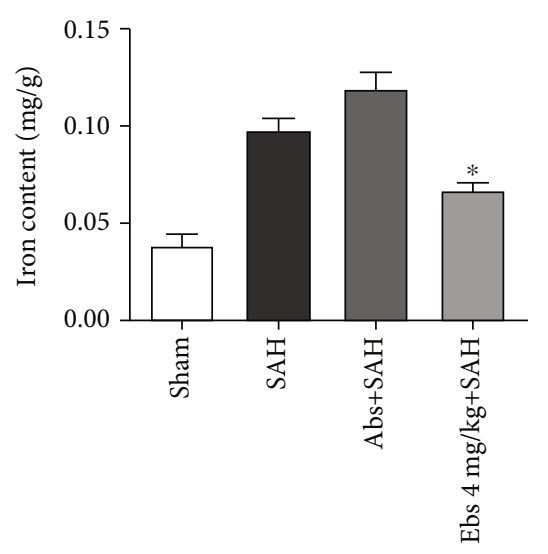

(k)

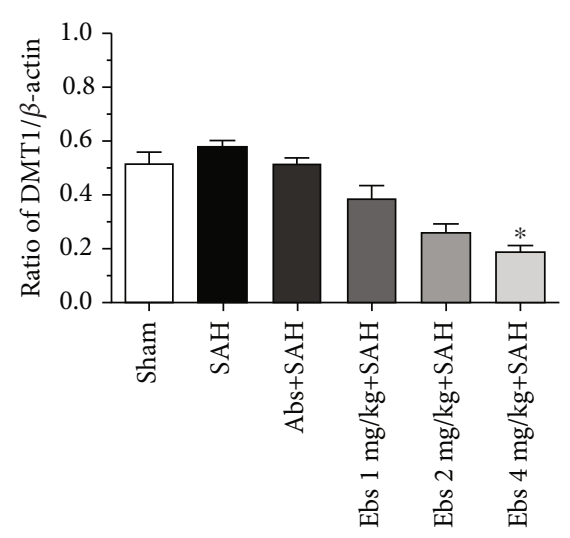

(h)
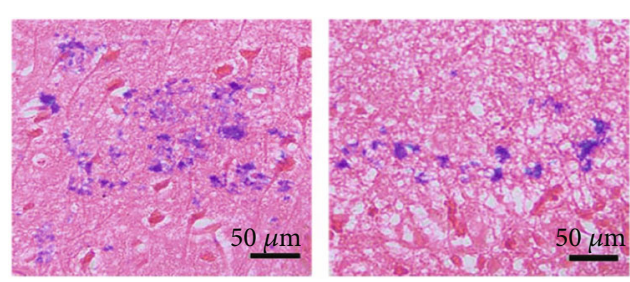

Ebs $4 \mathrm{mg} / \mathrm{kg}+\mathrm{SAH}$

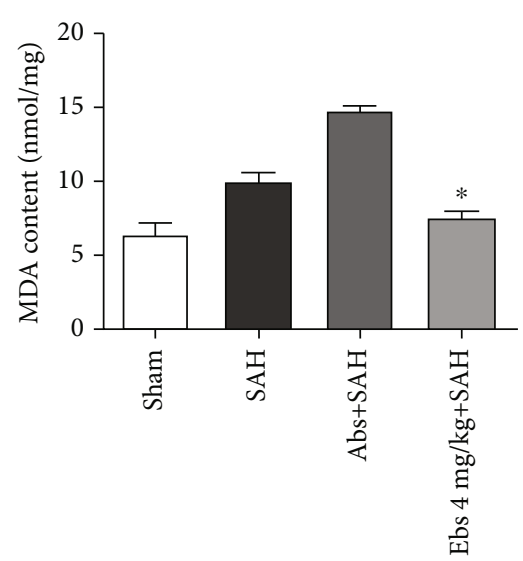

(l)

FIgURE 3: Continued. 


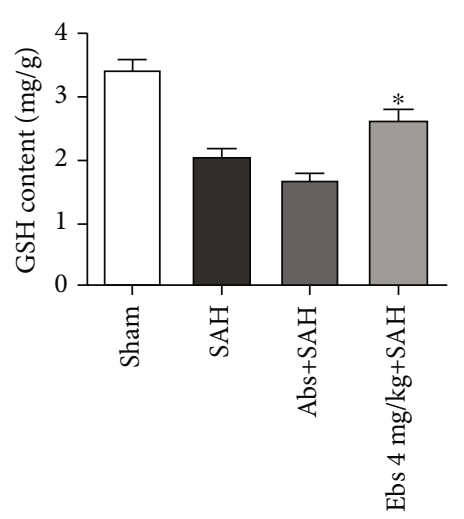

$(\mathrm{m})$

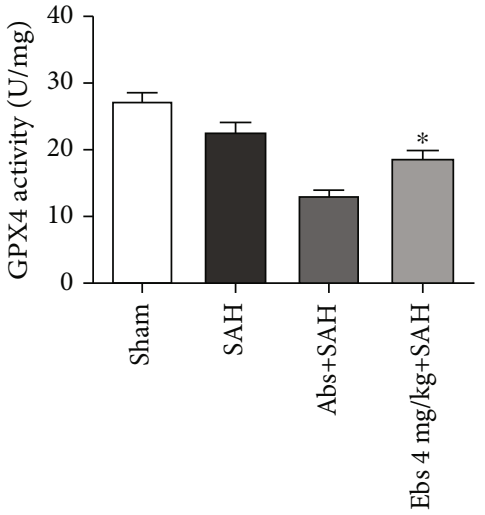

(n)

FIGURE 3: Immunofluorescence staining for hepcidin, DMT1 and GPX4 showed co-localization in neurons $24 \mathrm{~h}$ after SAH. Hepcidin, DMT1 and GPX4 were co-localized with NeuN-positive cells (neurons); hepcidin (red), DMT1 (red), GPX4 (red) NeuN (green), DAPI (nucleus, blue); original magnification $400 \times($ a) $(n=6)$. Expression of hepcidin, DMT1, FPN1 and GPX4 at different time points after SAH. Representative WBs of hepcidin, DMT1, FPN1 and GPX4 (b). Densitometric quantification of the optical densities of these protein bands (c-f). All protein expression levels were significantly changed at $24-72 \mathrm{~h}$ after SAH, $* P<0.05$ vs the sham group $(n=6$, each group). Expression of DMT1 and GPX4 after different doses of ebselen were introduced following SAH. Representative WBs of DMT1 and GPX4 (G), densitometric quantification of the optical densities of these protein bands (h-i), all protein expression levels were significantly changed at the dose of $4 \mathrm{mg} / \mathrm{kg}$ ebselen after SAH, $* P<0.05$ vs the Abs+SAH group, $(n=6$, each group). Perl's staining of iron after $4 \mathrm{mg} / \mathrm{kg}$ ebselen was administered. Positive areas $4 \mathrm{mg} / \mathrm{kg}$ ebselen decreased, compared with the Abs+SAH groups, final magnification $400 \times(\mathrm{j})(n=6$, each group). The activity of components of ferroptosis (iron content, MDA, GSH, and GPX4) were determined after $4 \mathrm{mg} / \mathrm{kg}$ ebselen was introduced. Treatment with $4 \mathrm{mg} / \mathrm{kg}$ ebselen decreased MDA while increasing GSH and GPX4 activity and protecting against ferroptosis, compared with the Abs+SAH groups $(\mathrm{k}-\mathrm{n}), * P<0.05$ vs the Abs+SAH group ( $n=6$, each group).

hepcidin and DMT1 both decreased, whereas FPN1 and GPX4 increased after heparin injection. Heparin $(1,2.5$ and $5 \mathrm{mg} / \mathrm{kg}$ ) decreased the expression of hepcidin, and the effect of $5 \mathrm{mg} / \mathrm{kg}$ heparin was most robust $(\mathrm{p}<0.05)(n=6$, Figure 6(b)), compared with the NS+SAH. DMT1 expression was significantly decreased with heparin at doses of $2.5 \mathrm{mg} / \mathrm{kg}$ and $5 \mathrm{mg} / \mathrm{kg}(\mathrm{p}<0.05)(n=6$, Figure $6(\mathrm{c}))$, compared with the NS + SAH. FPN1 expression was significantly increased with heparin at doses of $5 \mathrm{mg} / \mathrm{kg}(\mathrm{p}<0.05)(n=6$, Figure 6(d)), compared with the NS+SAH. GPX4 was significantly increased with heparin at a dose of $5 \mathrm{mg} / \mathrm{kg}$ $(\mathrm{p}<0.05)(n=6$, Figure 6(e)), compared with the NS + SAH groups. Heparin at $5 \mathrm{mg} / \mathrm{kg}$ had a significant effect on the expression of hepcidin, DMT1, FPN1 and GPX4 $(n=6$, Figure 6(a)).

Out of heparin doses, $5 \mathrm{mg} / \mathrm{kg}$ heparin was administered to evaluate iron metabolism, ferroptosis and EBI. The results indicated that $5 \mathrm{mg} / \mathrm{kg}$ heparin decreased the Perl's iron staining and iron content $(\mathrm{p}<0.05)$ (Figures 6(f), 6(g)), compared with the NS + SAH groups. It also decreased the MDA content and increased the GSH content and GPX4 activity $(\mathrm{p}<0.05)(n=6$, Figures 6(h), 6(i), 6(j)), compared with the $\mathrm{NS}+\mathrm{SAH}$ groups. Additionally, $5 \mathrm{mg} / \mathrm{kg}$ heparin protected against ferroptosis, which reduced the size of mitochondria in TEM after SAH $(n=6$, Figure 4(i)). It also improved the neurological deficits, reduced the brain edema, and decreased the blood-brain barrier permeability, thus mitigating EBI $(\mathrm{p}<0.05)(n=6$, Figures $5(\mathrm{f}), 5(\mathrm{~g}), 5(\mathrm{~h}))$, compared with the NS + SAH groups.
3.4. Hepcidin Stimulation Promoted the Expression of DMT1, Influenced Iron Metabolism, and Aggravated EBI and Ferroptosis after SAH. An inducer of hepcidin, OSM, was used to verify the role of hepcidin in SAH-induced brain injury. OSM was administered (100 ng, $1 \mu \mathrm{g}, 5 \mu \mathrm{g}$, and $10 \mu \mathrm{g}$ per rat) $24 \mathrm{~h}$ before SAH. The Western blot results indicated that OSM enhanced the expression of hepcidin and DMT1, whereas GPX4 expression was reduced. OSM induced the expression of hepcidin, while $10 \mu \mathrm{g}$ OSM even more potently stimulated the expression of hepcidin $(\mathrm{p}<0.05)(n=6$, Figure 6(l)), in comparison with the PBS + SAH groups. OSM at doses of $1 \mu \mathrm{g}, 5 \mu \mathrm{g}$ and $10 \mu \mathrm{g}$ significantly stimulated the expression of DMT1 $(\mathrm{p}<0.05)(n=6$, Figure $6(\mathrm{~m})$ ), as compared to the PBS $+\mathrm{SAH}$ groups. OSM at doses of $10 \mu \mathrm{g}$ significantly inhibited the expression of FPN1 $(\mathrm{p}<0.05)(n=6$, Figure $6(\mathrm{n}))$, as compared to the PBS + SAH groups. OSM at doses of $100 \mathrm{ng}, 1 \mu \mathrm{g}, 5 \mu \mathrm{g}$, and $10 \mu \mathrm{g}$ per rat decreased the expression of GPX4, whereas $10 \mu \mathrm{g}$ OSM very potently alleviated the expression of GPX4 $(\mathrm{p}<0.05)(n=6$, Figure 6(o)), in contrast to the PBS $+\mathrm{SAH}$ groups. Thus, OSM at a dose of $10 \mu \mathrm{g}$ had a very significant effect on the expression of hepcidin, FPN1, DMT1 and GPX4 as well $(n=6$, Figure $6(\mathrm{k}))$.

Therefore, OSM at a dose of $10 \mu \mathrm{g}$ per rat was employed to detect the components of ferroptosis. OSM at $10 \mu \mathrm{g}$ promoted iron metabolism and ferroptosis, by increasing and Perl's iron staining and the iron content $(\mathrm{p}<0.05)(n=6$, Figures $6(\mathrm{p})$, $6(\mathrm{q})$ ), in comparison with the PBS $+\mathrm{SAH}$ groups. It also increased the MDA content, decreased the GSH content and 


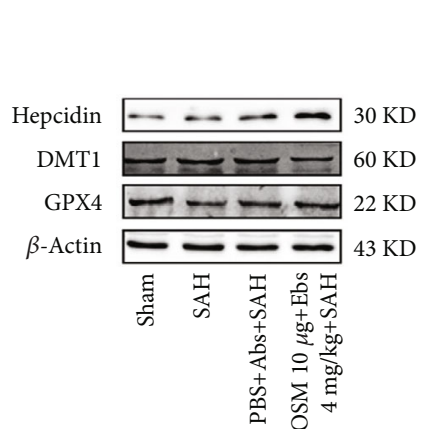

(a)

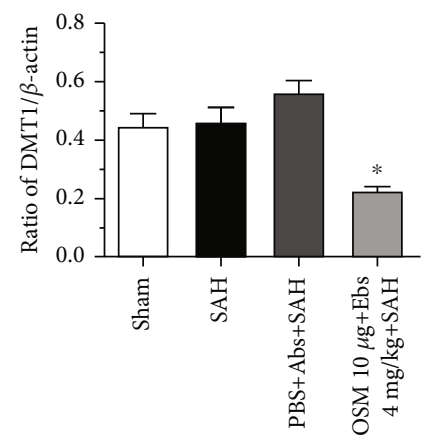

(c)

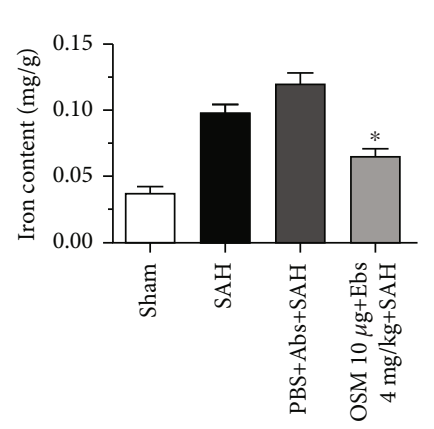

(e)

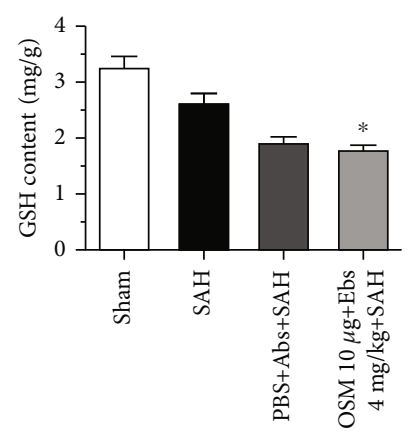

(g)

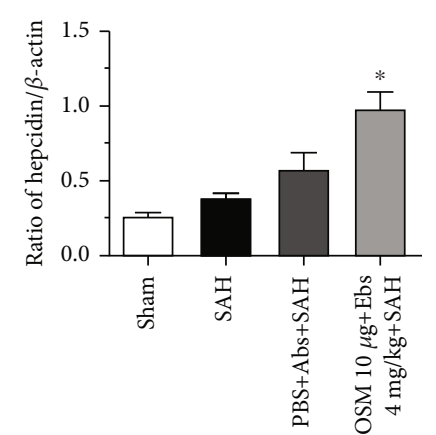

(b)

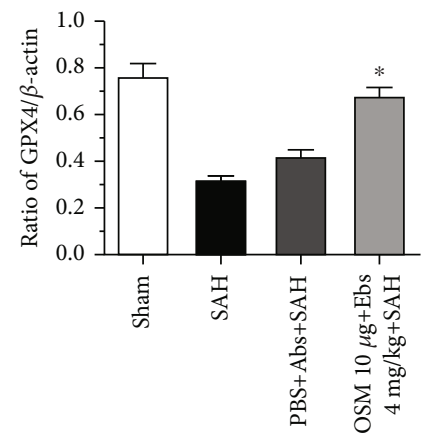

(d)

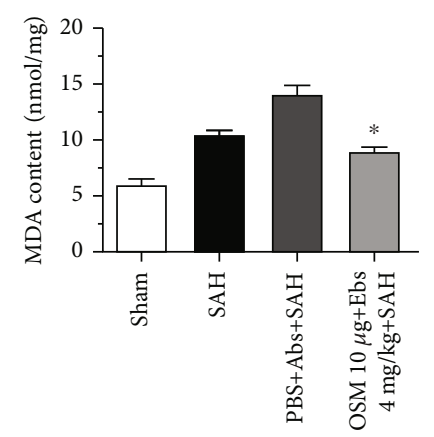

(f)

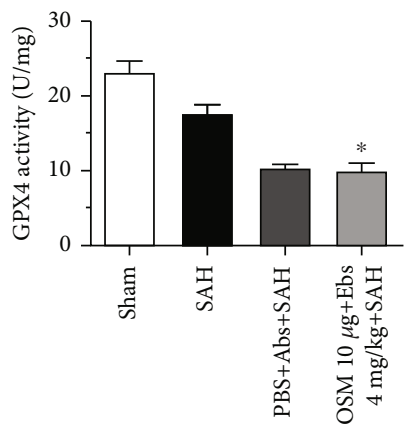

(h)

Figure 4: Continued. 


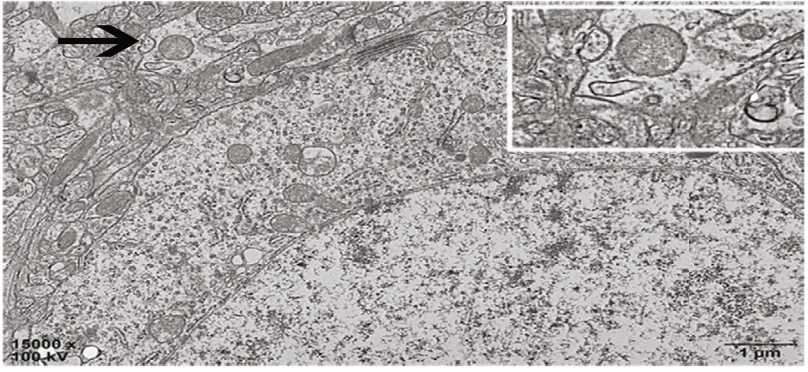

$\mathrm{Abs}+\mathrm{SAH}$

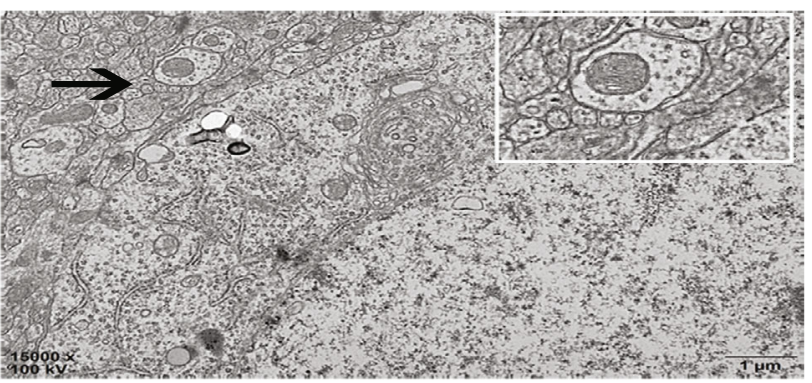

$\mathrm{NS}+\mathrm{SAH}$

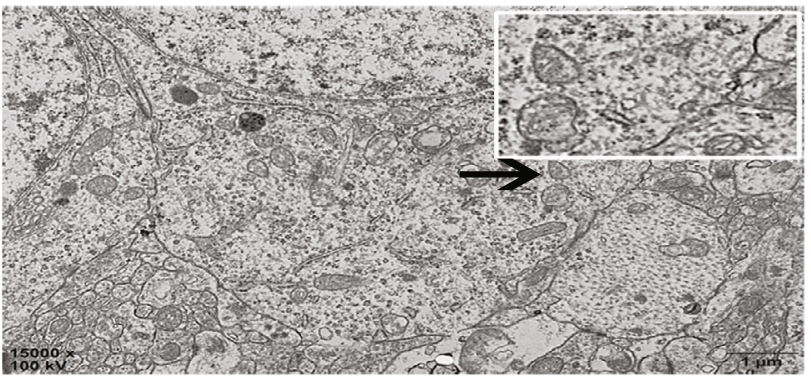

$\mathrm{PBS}+\mathrm{SAH}$

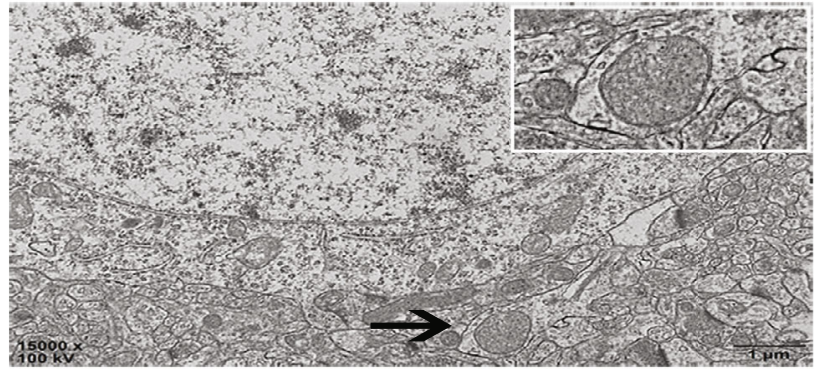

Ebs $4 \mathrm{mg} / \mathrm{kg}+\mathrm{SAH}$

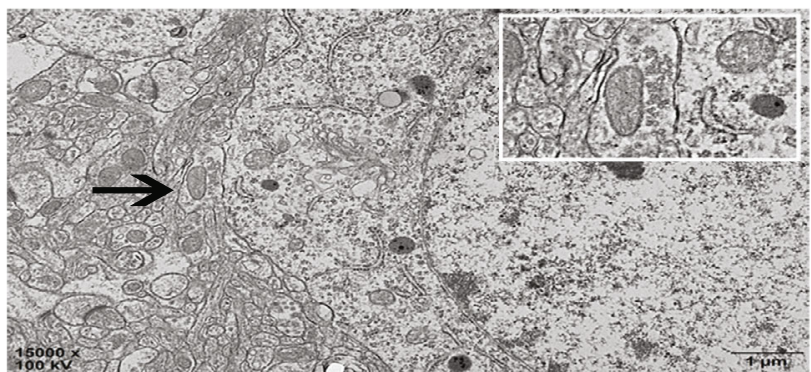

Hep $5 \mathrm{mg} / \mathrm{kg}+\mathrm{SAH}$

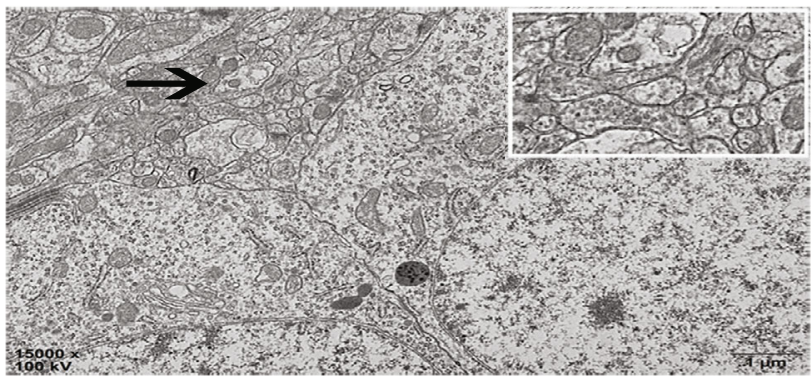

OSM $10 \mu \mathrm{g}+\mathrm{SAH}$

(i)

FIGURE 4: Both OSM and ebselen were used, the expression of hepcidin was improved (b), DMT1 was reduced (c), while GPX4 was increased (d), $* P<0.05$ vs PBS $+\mathrm{SAH}$ group ( $n=6$, each group). $4 \mathrm{mg} / \mathrm{kg}$ ebselen could reverse the effects of $10 \mu \mathrm{g}$ OSM on iron metabolism and ferroptosis $(\mathrm{e}-\mathrm{h}), * P<0.05$ vs $\mathrm{PBS}+\mathrm{SAH}$ group $(n=6$, each group). $5 \mathrm{mg} / \mathrm{kg}$ heparin protected against ferroptosis, which reduced size of mitochondria in TEM after SAH, compared to the NS + SAH; $4 \mathrm{mg} / \mathrm{kg}$ ebselen decreased the size of mitochondria of ferroptosis after $\mathrm{SAH}$, and it protected against ferroptosis, compared to the Abs+SAH; OSM $10 \mu$ g aggravated ferroptosis, which augmented the size of mitochondria in TEM after SAH, compared to the PBS + SAH (i) ( $n=6$, each group).

GPX4 activity $(\mathrm{p}<0.05)(n=6$, Figures $6(\mathrm{r}), 6(\mathrm{~s}), 6(\mathrm{t}))$, in comparison with the PBS $+\mathrm{SAH}$. Additionally, OSM $10 \mu \mathrm{g}$ aggravated ferroptosis, which augmented the size of mitochondria in TEM after SAH $(n=6$, Figure 5(i)), in comparison with the PBS + SAH. $10 \mu \mathrm{g}$ OSM also decreased the neurological deficits, increased the brain edema, and increased the bloodbrain barrier permeability, thus aggravated EBI $(\mathrm{p}<0.05)$ $(n=6$, Figure 6(i), 6(j), 6(k)), compared with the NS $+\mathrm{SAH}$ groups.

3.5. Induction of Hepcidin Promoted Iron Metabolism Enhancement and Ferroptosis, Was Reversed via DMT1 Inhibition in SAH Rats. The effects of OSM and ebselen on hepcidin and DMT1 were revealed as above,10 $\mu \mathrm{g}$ OSM and $4 \mathrm{mg} / \mathrm{kg}$ ebselen were both employed to validate the effects of hepcidin in SAH $(n=6$, Figure $4(a))$. With this combined treatment, Western blot results indicated that the expression of hepcidin was improved $(\mathrm{p}<0.05)(n=6$, Figure 4(b)). Moreover, DMT1 was reduced while GPX4 was increased $(p<0.05)(n=6$, Figures $4(c), 4(d))$, as compared with the $\mathrm{PBS}+\mathrm{Abs}+\mathrm{SAH}$ groups. According to the results presented above, $10 \mu \mathrm{g}$ OSM elevated the iron content, when it was used alone in SAH. Whereas $4 \mathrm{mg} / \mathrm{kg}$ ebselen could reverse the effects of $10 \mu \mathrm{g}$ on iron content, when both OSM and ebselen were used, compared with the PBS + Abs+SAH groups. Therefore, $4 \mathrm{mg} / \mathrm{kg}$ ebselen could reverse the effects of $10 \mu \mathrm{g}$ OSM on iron metabolism and ferroptosis $(n=6$, Figures $4(\mathrm{e}), 4(\mathrm{f}), 4(\mathrm{~g}), 4(\mathrm{~h}))$.

3.6. SAH Severity and Mortality. SAH grade and mortality are prognostic indicators, which recorded after surgery and treatment. The SAH grading scores were similar among each 


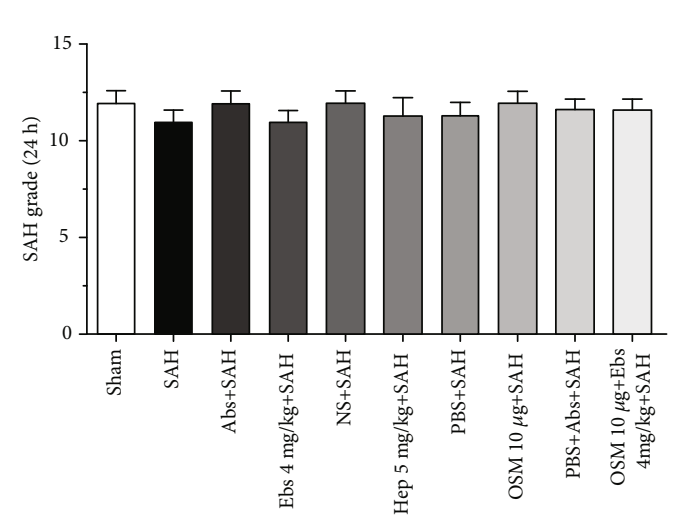

(a)

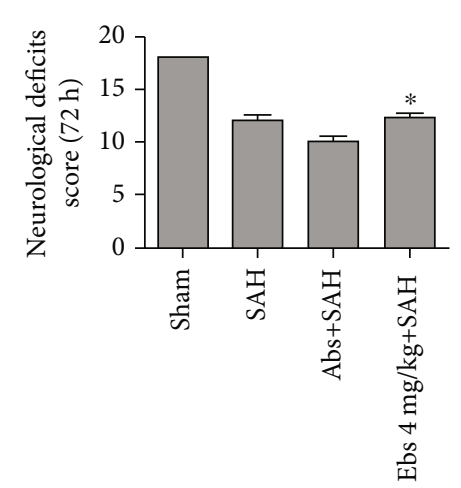

(c)

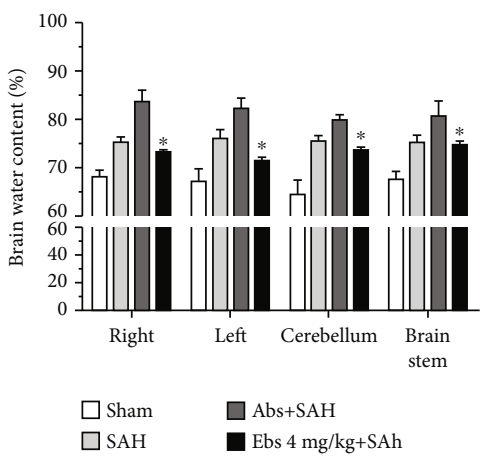

(e)

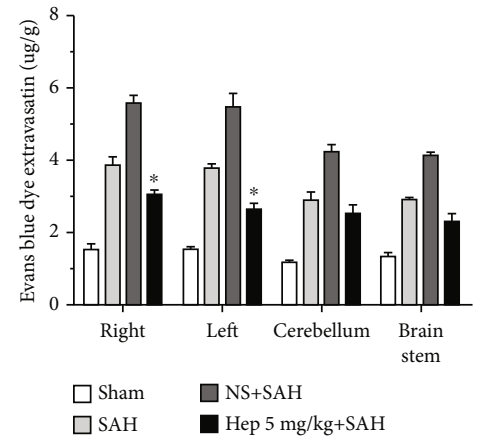

(g)

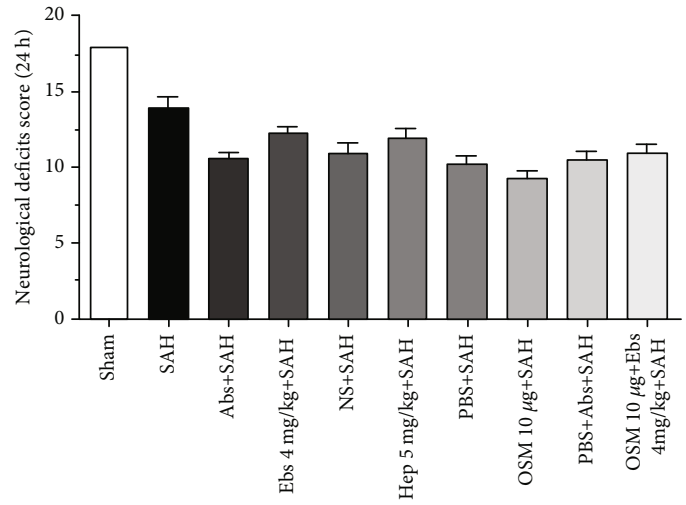

(b)

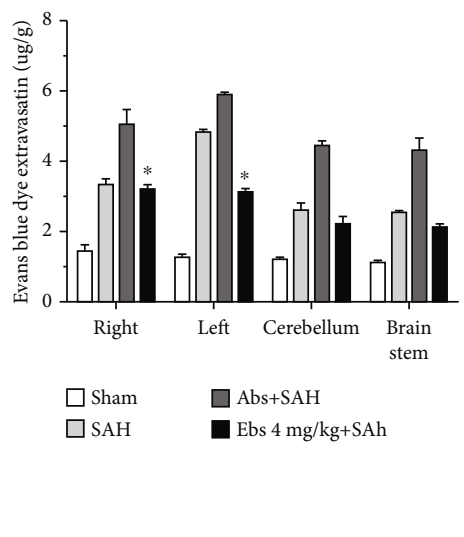

(d)

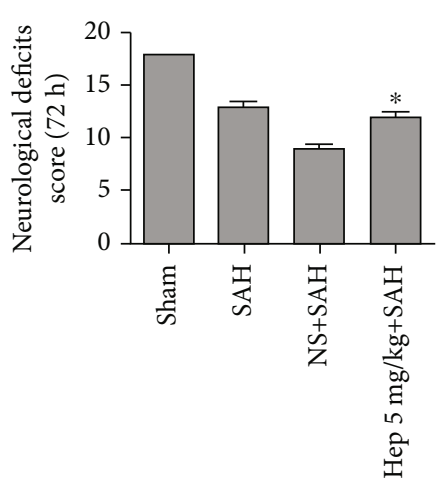

(f)

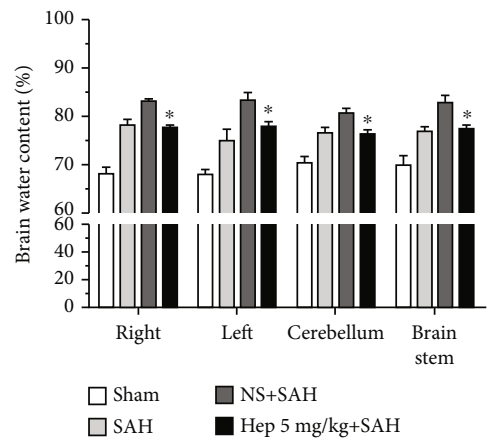

(h)

FIgURE 5: Continued. 


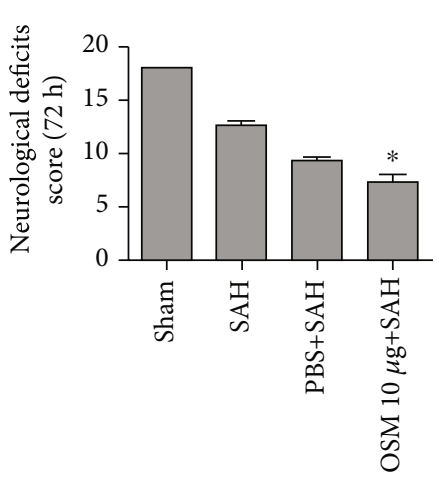

(i)

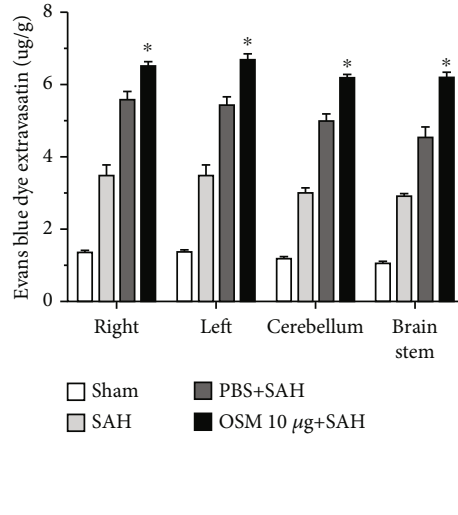

(j)

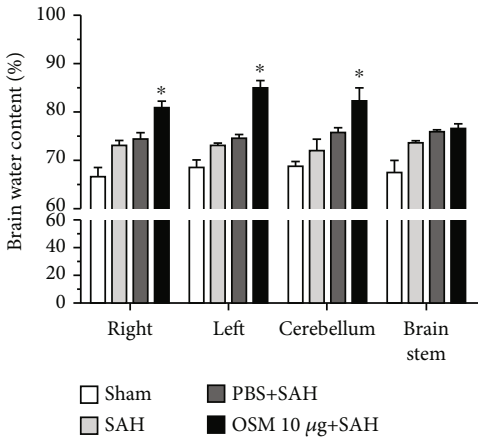

$(\mathrm{k})$

Figure 5: The SAH grading scores were similar among each surgery group ( $n=6$, Fig. 4(a), $\mathrm{p}=0.852)$, The neurological deficit scores among each surgery group $(n=6$, Fig. $4(\mathrm{~b}), \mathrm{p}=0.806)$ along with the evaluation of SAH grade. Treatment with $4 \mathrm{mg} / \mathrm{kg}$ ebselen to determine the EBI (c-e), $4 \mathrm{mg} / \mathrm{kg}$ ebselen decreased neurological function deficit compared with the SAH and Abs+SAH groups (c). BBB permeability was also alleviated in four areas ( $\mathrm{LH}, \mathrm{RH}, \mathrm{CB}$, and BS) after treatment with $4 \mathrm{mg} / \mathrm{kg}$ ebselen (d). Brain water content was decreased in four areas (LH, $\mathrm{RH}, \mathrm{CB}$, and BS) after treatment with $4 \mathrm{mg} / \mathrm{kg}$ ebselen (e), $* P<0.05$ vs Abs $+\mathrm{SAH}$ group ( $n=6$, each group). Treatment with $5 \mathrm{mg} / \mathrm{kg}$ heparin to determine the EBI (f-h), $5 \mathrm{mg} / \mathrm{kg}$ heparin decreased neurological functions (f). BBB permeability based on Evans blue staining in the LH and RH was decreased by the $5 \mathrm{mg} / \mathrm{kg}$ heparin treatment $(\mathrm{g})$. Brain water content was decreased in four areas (LH, RH, CB, and BS) after treatment with $5 \mathrm{mg} / \mathrm{kg}$ heparin (h), $* P<0.05$ vs NS $+\mathrm{SAH}$ group $(n=6$, each group). Treatment with $10 \mu \mathrm{g}$ OSM to determine the EBI (i-k), $10 \mu \mathrm{g}$ OSM aggravated neurological functions (i). BBB permeability based on Evans blue staining in the LH and $\mathrm{RH}$ was increased by the $10 \mu \mathrm{g}$ OSM treatment $(\mathrm{j})$. Brain water content was increased in four areas (LH, RH, CB, and BS) after treatment with $10 \mu \mathrm{g}$ OSM $(\mathrm{k}), * P<0.05$ vs $\mathrm{PBS}+\mathrm{SAH}$ group $(n=6$, each group).

surgery group ( $n=6$ Fig. $5(\mathrm{a}), \mathrm{p}=0.852$ ), The neurological deficit scores among each surgery group $(n=6$, Fig. $5(\mathrm{~b})$, $\mathrm{p}=0.806$ ), along with the evaluation of $\mathrm{SAH}$ grade. A total of 30 rats died during or after model constructed due to severe $\mathrm{SAH}$, and another 21 rats were excluded because of their low SAH grade. No death was observed in the sham group, after surgery, with mortality rates (Table 1), which were calculated as follows: sham $=0 \%$ (0 of 40$), \mathrm{SAH}=$ $27.27 \%$ (18 of 66$), \mathrm{NS}=25.0 \%$ (6 of 24$), \mathrm{Hep}=16.67 \%$ (6 of 36$), \mathrm{Abs}=17.39 \%$ (4 of 23 ), $\mathrm{Ebs}=17.65 \%$ (9 of 51 ), $\mathrm{PBS}=26.92 \%(7$ of 26$), \mathrm{OSM}=21.28 \%(10$ of 47$)$ and PBS $+\mathrm{Abs}=25 \%$ (4 of 16), OSM + Ebs $=29.17 \%$ (7 of 24). However, there were no statistically significant differences in mortality rates between treatment groups after analysis with a Fisher two-sided exact test.

\section{Discussion}

EBI after SAH remains a major cause of high lethality and disability of patients, and previous studies have verified that iron metabolism disturbances play an important role in $\mathrm{SAH}$ [29]. Ferroptotic cell death is morphologically, biochemically and genetically distinct from apoptosis, various forms of necrosis, and autophagy [30,31] The process of ferroptosis is characterized by the overwhelming, iron-dependent lethal accumulation of lipid ROS, and unlike other forms of apoptotic and nonapoptotic death, this requirement for ROS accumulation appears to be indispensable in ferroptosis [32]. Dixon et al. first explored the mechanism of ferroptosis, which is dependent on intracellular iron and the accu- mulation of LPO [5]. The main observed morphological effect was a decrease in the size of mitochondria, whereas the iron content and LPO products, also known as lipid ROS, increased to induce ferroptosis, accompanied by a decreased GSH content and GPX4 activity [9, 33]. Exogenous iron could boost the components of ferroptosis and morphological aspects of cell death, as previously described, and it is believed that disturbed iron metabolism is primarily responsible for ferroptosis $[34,35]$. The cerebral occurrence of ferroptosis has been observed in Parkinson's disease, and may occur in several types of brain injury [36], as well as in infection [37] and cancer [38, 39]. Ferroptosis has been found occur in EBI after SAH, lines reported that via reduction of lipid peroxidation and inhibiting p53 could alleviate ferroptosis in EBI after SAH [40]. Notably, ferroptosis strongly relates to iron metabolism but whether influencing iron metabolism could alleviate ferroptosis and the pathway have not been reported.

Evidence has indicated that the only intracellular iron transporter, DMT1, may mediate intracellular iron metabolism [41], and be involved in ferroptosis induction, while inhibiting DMT1 by ebselen may modulate the iron uptake [18]. Moreover, hepcidin is a major iron metabolism hormone and may influence the iron content in the ischemic brain, and lines reported that hepcidin could increase DMT1 and inhibit FPN1 expression in regulating iron metabolism $[6,42]$. Recently, several lines of evidence have shown that recombinant hepcidin could stimulate the expression of DMT1, and suppressing hepcidin may decrease brain iron content $[43,44]$. Thus, we hypothesized 


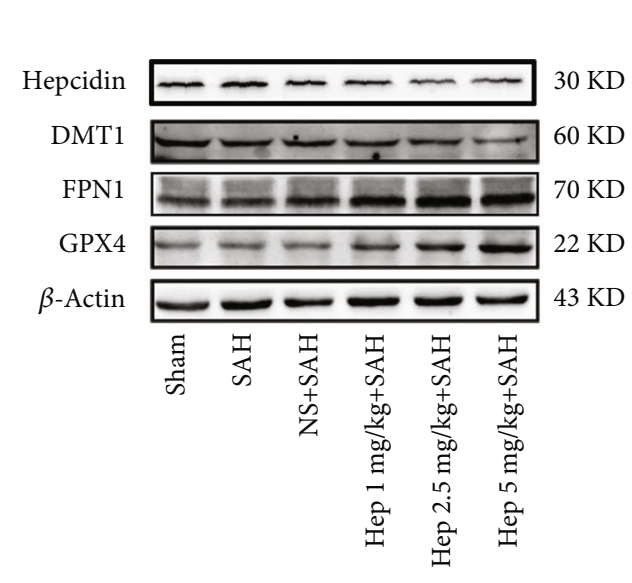

(a)

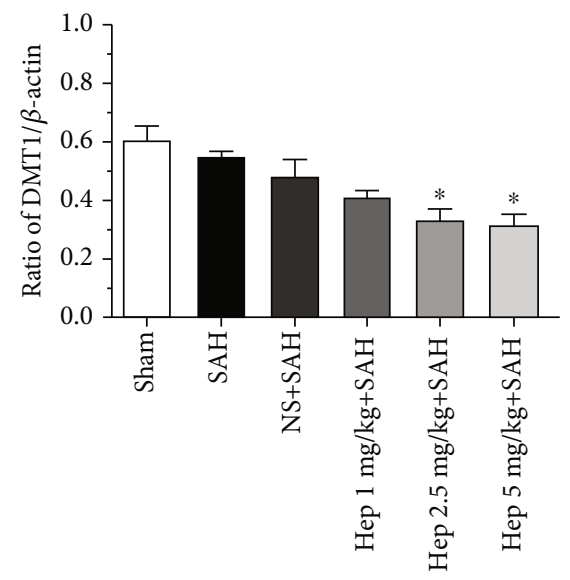

(c)

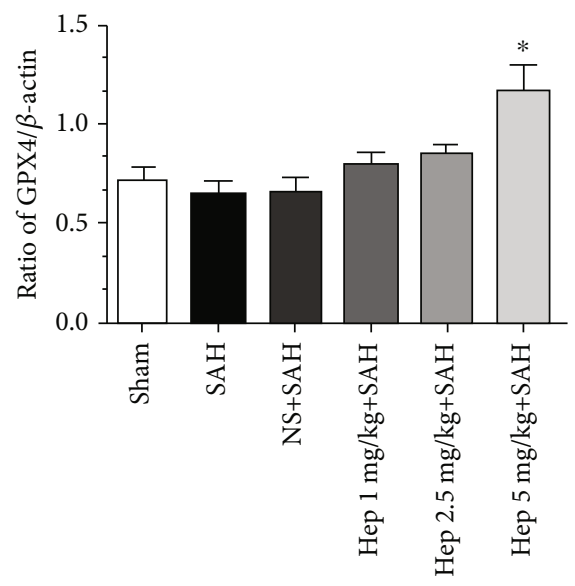

(e)

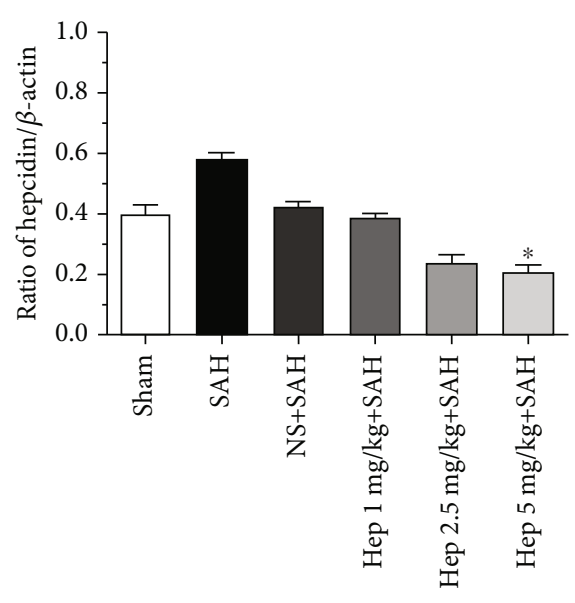

(b)

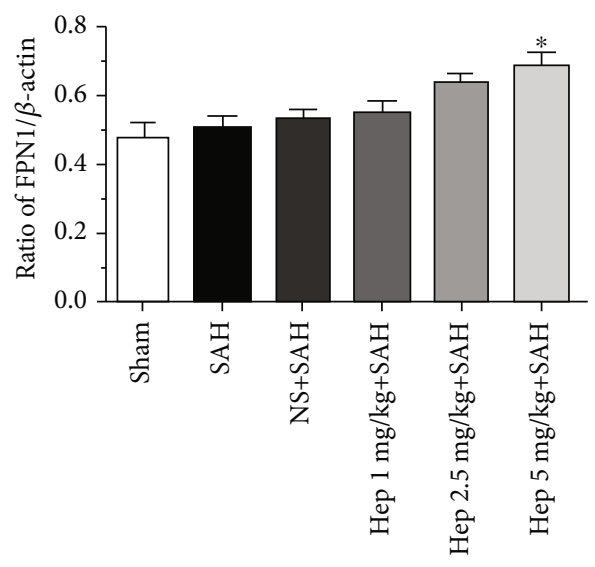

(d)
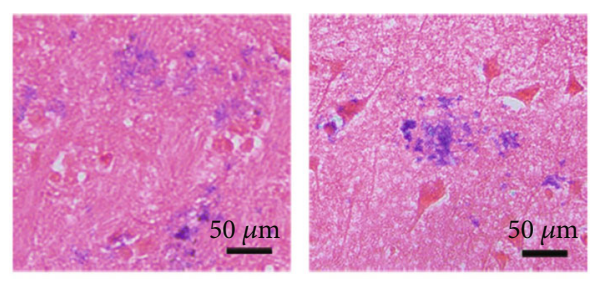

Hep 5 mg/kg+SAH

(f)

FIGURE 6: Continued. 


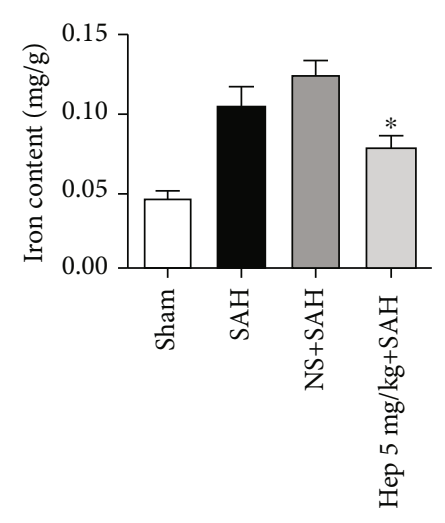

(g)

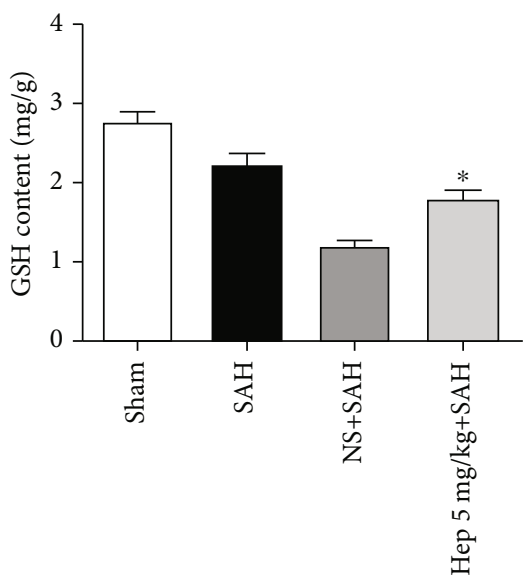

(i)

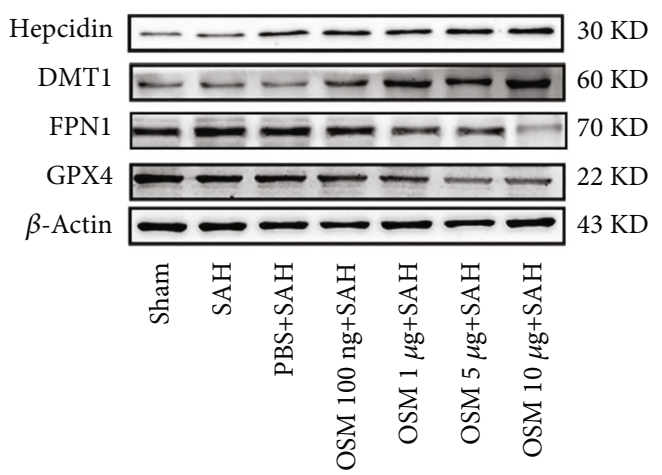

(k)

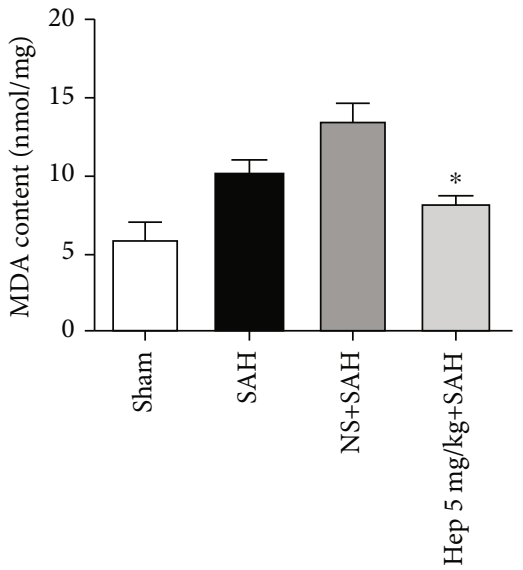

(h)

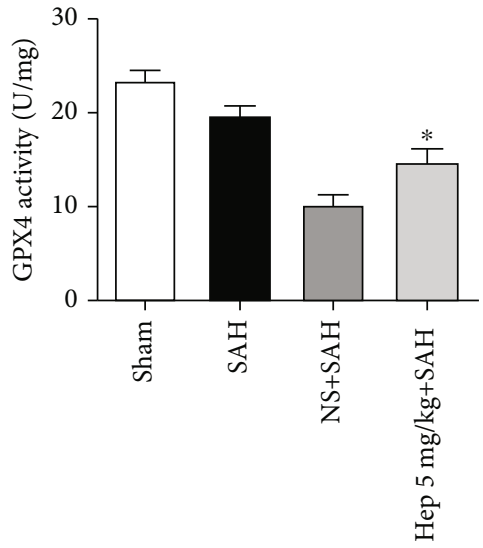

(j)

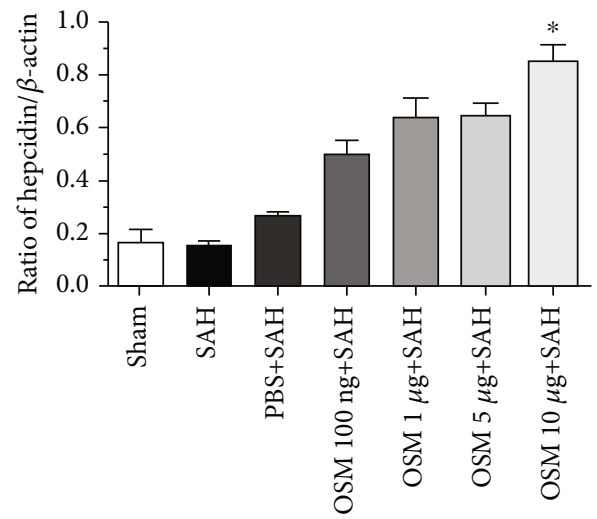

(1)

Figure 6: Continued. 


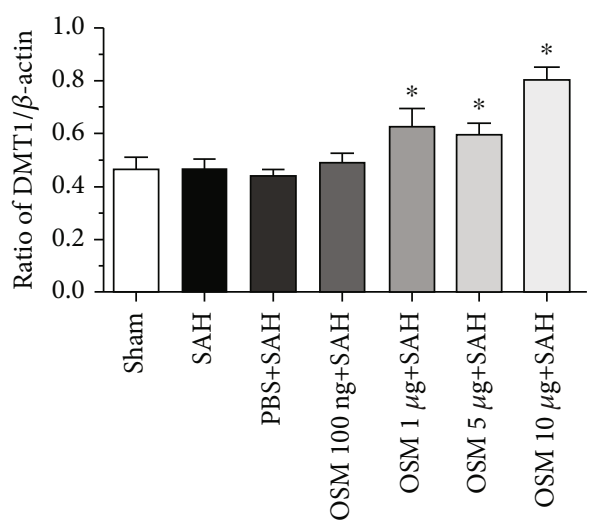

(m)

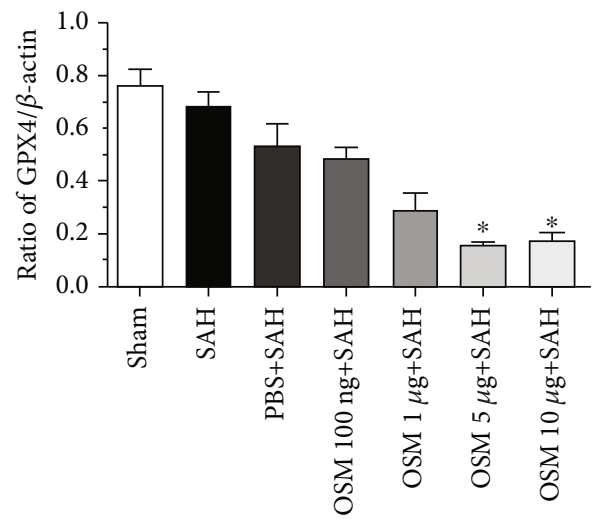

(o)

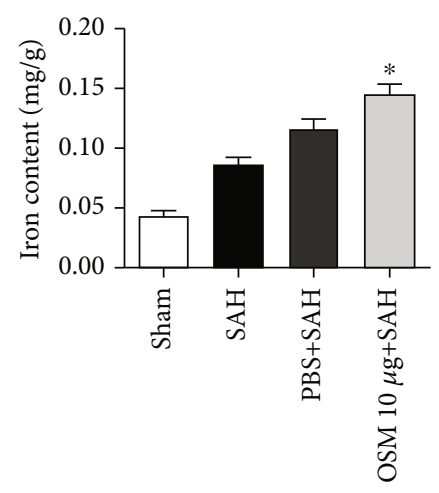

(q)

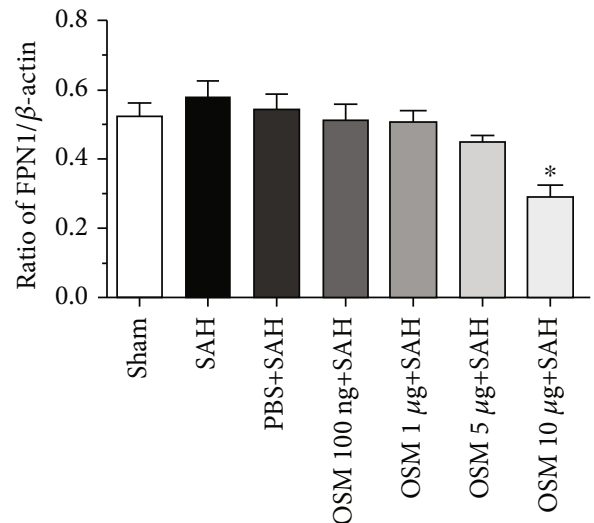

(n)

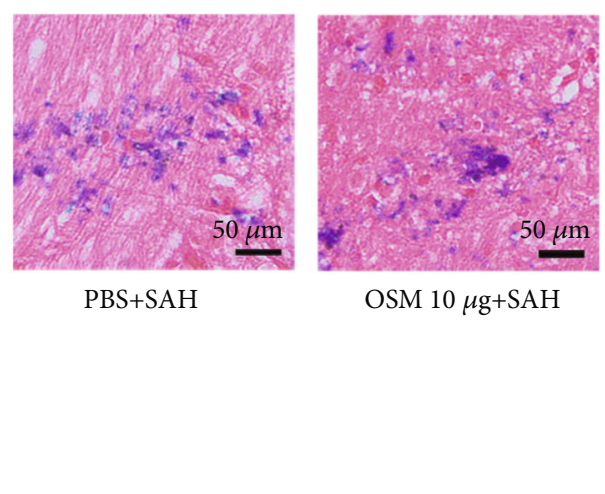

(p)

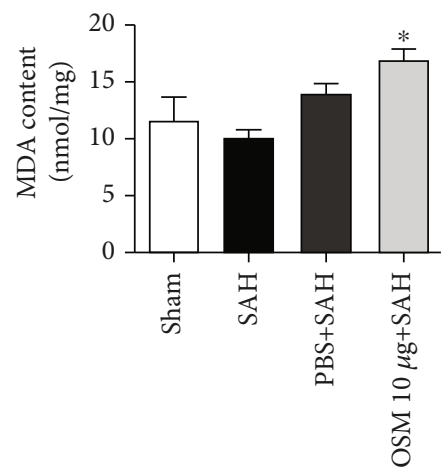

(r)

Figure 6: Continued. 


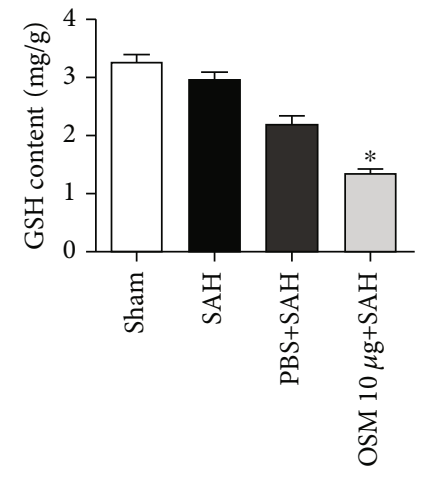

(s)

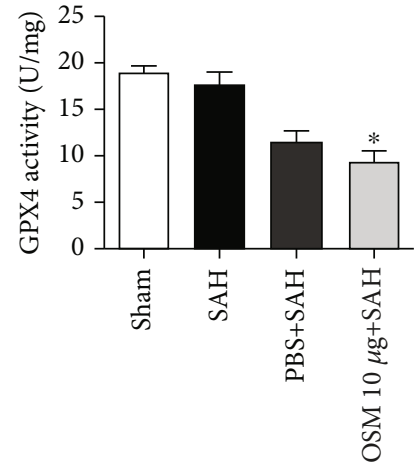

(t)

Figure 6: Expression of hepcidin, DMT1, FPN1 and GPX4 after different doses of heparin were introduced following SAH. Representative WBs of hepcidin, DMT1, FPN1 and GPX4 (a), densitometric quantification of the optical densities of these protein bands (b-e), all protein expression levels were significantly changed at the dose of $5 \mathrm{mg} / \mathrm{kg}$ heparin after $\mathrm{SAH},{ }^{*} \mathrm{P}<0.05$ vs the NS $+\mathrm{SAH}$ group ( $n=6$, each group). Perl's iron staining after $5 \mathrm{mg} / \mathrm{kg}$ heparin was administered. Positive areas $5 \mathrm{mg} / \mathrm{kg}$ heparin decreased, compared with the NS + SAH groups, final magnification $400 \times$ (f). The activity of components of ferroptosis (iron content, MDA, GSH, and GPX4) were determined after $5 \mathrm{mg} / \mathrm{kg}$ heparin was introduced. Treatment with $5 \mathrm{mg} / \mathrm{kg}$ heparin decreased iron content and MDA, while increasing GSH and GPX4 activity and protecting against ferroptosis, compared with the NS + SAH groups (g-j), ${ }^{*} P<0.05$ vs the NS + SAH group $(n=6$, each group). Expression of hepcidin, DMT1, FPN1, and GPX4 after different doses of OSM were introduced following SAH. Representative WBs of hepcidin, DMT1, FPN1, and GPX4 (k), densitometric quantification of the optical densities of these protein bands (l-o), and all protein expression levels were significantly changed at the dose of $10 \mu \mathrm{g}$ OSM after SAH, ${ }^{*} P<0.05$ vs. the NS+SAH group ( $n=6$, each group). Perl's iron staining after $10 \mu \mathrm{g}$ OSM was administered. Positive areas $10 \mu \mathrm{g}$ OSM increased, compared with the PBS+SAH groups, final magnification $\times 400(\mathrm{p})$. The activity of components of ferroptosis (iron content, MDA, GSH, and GPX4) were determined after $10 \mu \mathrm{g}$ OSM was introduced. Treatment with $10 \mu \mathrm{g}$ OSM increased iron content and MDA, while decreasing GSH and GPX4 activity and aggravating ferroptosis, compared with the PBS+SAH groups (q-t), ${ }^{*} P<0.05$ vs. the PBS+SAH group ( $n=6$, each group).

that hepcidin might have an effect on iron metabolism and ferroptosis, via DMT1 activation, in EBI after SAH (Figure 1).

To validate the effect of hepcidin in $\mathrm{SAH}$, we examined the expression of hepcidin and DMT1, FPN1 over time in EBI after SAH, and the results showed that both hepcidin and DMT1 increased, FPN1 decreased. Whereas GPX4, which serves as the main regulator of ferroptosis, decreased compared with the sham group. These findings are consistent with our previous study, which demonstrated that SAH stimulates the expression of hepcidin. Yang et al. found that cancer cell death occurred in the form of ferroptosis, in which GPX4 decreased [42]. In our previous investigation, we also determined that the expression of GPX4, the protein protective against ferroptosis decreased in SAH. These findings prompted our hypothesis that SAH might cause cell death in the form of ferroptosis.

In this study, we obtained several fruitful results as follows. Firstly, the iron metabolism related proteins, hepcidin and DMT1 were upregulated, while, FPN1 decreased, at the same time, the GPX4 also decreased in EBI after SAH. Secondly, the only intracellular iron transporter, DMT1, has been suggested to induce intracellular iron release and lead to iron deposition, at last induced ferroptosis. An inhibitor of DMT1, ebselen, was employed to verify the effects of DMT1 on iron metabolism and ferroptosis following SAH. The findings revealed that ebselen could decrease the iron content and Perl's iron staining and alleviate ferroptosis in SAH. Thirdly, an inhibitor and an inducer of hepcidin were administered to detect the effects of hepcidin on iron metab- olism and ferroptosis in EBI after SAH. The findings proved that the inhibitor of hepcidin, heparin, could decrease DMT1, increase FPN1, also alleviate the iron content, Perl's iron staining, and ferroptosis in $\mathrm{SAH}$, consistent with the effect of ebselen on DMT1; moreover, heparin could also protect against EBI. The results suggested that the inducer of hepcidin, OSM, could increase DMT1, decrease FPN1, also elevate the iron content, enhance Perl's iron staining, and aggravate ferroptosis and EBI. Finally, the results suggested that hepcidin could influence iron metabolism through DMT1, induce iron accumulation, promote LPO accumulation, and then lead to ferroptosis. The inducer of hepcidin and the inhibitor of DMT1, OSM and ebselen, respectively, were both used to investigate the effects of hepcidin on metabolism and ferroptosis via DMT1 in EBI after $\mathrm{SAH}$. The results suggested that ebselen could reverse the effects of OSM and hepcidin on metabolism and ferroptosis.

However, several shortcomings of this study need to be discussed. Different doses of OSM, heparin and ebselen were administered in this research, however, the safety of these doses of drugs remains to be verified. Although gradient concentrations of OSM, heparin and ebselen were introduced in this research, only the most significant influences of the doses of OSM, heparin and ebselen on various protein levels were adopted to investigate ferroptosis and EBI. Other doses of OSM, heparin and ebselen need to be investigated to confirm that OSM, heparin and ebselen have conclusive impacts on ferroptosis and EBI after SAH. However, it needs to be stressed that this study still examined many doses dependent effects, which is not always the case regarding 
TABLE 1: SAH grade and mortality are prognostic indicators, which recorded after surgery and treatment. A total of 30 rats died during or after model constructed due to severe SAH, and another 21 rats were excluded because of their low SAH grade. No death was observed in the sham group, after surgery, with mortality rates (Table 1), which were calculated as follows: sham $=0 \%(0$ of 40$), \mathrm{SAH}=27.27 \%(18$ of 66), $\mathrm{NS}=25.0 \%$ (6 of 24), Hep $=16.67 \%$ (6 of 36), Abs=17.39\% (4 of 23), Ebs=17.65\% (9 of 51), PBS=26.92\% (7 of 26), $\mathrm{OSM}=21.28 \%$ (10 of 47$)$ and $\mathrm{PBS}+\mathrm{Abs}=25 \%$ (4 of 16$), \mathrm{OSM}+\mathrm{Ebs}=29.17 \%$ (7 of 24$)$. However, there were no statistically significant differences in mortality rates between treatment groups.

\begin{tabular}{|c|c|c|c|c|c|}
\hline Groups & Mortality & Excluded & Groups & Mortality & Excluded \\
\hline Experiment 1 & & & Experiment 2 & & \\
\hline Sham & $0 \%(0 / 8)$ & 0 & Sham & $0 \%(0 / 8)$ & 0 \\
\hline SAH ( 6 h 12 h, 24 h, 48 h, 72 h) & $21.8 \%(7 / 32)$ & 3 & SAH & $37.5 \%(3 / 8)$ & 1 \\
\hline Experiment 3 & & & $\mathrm{SAH}+\mathrm{Abs}$ & $20 \%(2 / 10)$ & 0 \\
\hline Sham & $0 \%(0 / 8)$ & 0 & $\mathrm{SAH}+\mathrm{Ebs}(1 \mathrm{mg} / \mathrm{kg})$ & $16.67 \%(2 / 12)$ & 1 \\
\hline SAH & $37.5 \%(3 / 8)$ & 1 & $\mathrm{SAH}+\mathrm{Ebs}(2 \mathrm{mg} / \mathrm{kg})$ & $23.07(3 / 13)$ & 1 \\
\hline $\mathrm{SAH}+\mathrm{NS}$ & $20 \%(2 / 10)$ & 0 & $\mathrm{SAH}+\mathrm{Ebs}(4 \mathrm{mg} / \mathrm{kg})$ & $20 \%(3 / 15)$ & 0 \\
\hline $\mathrm{SAH}+\mathrm{Hep}(1 \mathrm{mg} / \mathrm{kg})$ & $12.5 \%(1 / 8)$ & 1 & Experiment 5 & & \\
\hline $\mathrm{SAH}+\mathrm{Hep}(2.5 \mathrm{mg} / \mathrm{kg})$ & $22.22(2 / 9)$ & 1 & Sham & - & 0 \\
\hline $\mathrm{SAH}+\mathrm{Hep}(5 \mathrm{mg} / \mathrm{kg})$ & $11.1 \%(1 / 9)$ & 0 & SAH & - & 1 \\
\hline Experiment 4 & & & $\mathrm{SAH}+\mathrm{PBS}+\mathrm{Abs}$ & $25 \%(4 / 16)$ & 1 \\
\hline Sham & $0 \%(0 / 8)$ & 0 & $\mathrm{SAH}+\mathrm{OSM}(10 \mu \mathrm{g})+\mathrm{Ebs}(4 \mathrm{mg} / \mathrm{kg})$ & $29.1 \%(7 / 24)$ & \\
\hline SAH & $25 \%(2 / 8)$ & 2 & Experiment 6 & & 0 \\
\hline $\mathrm{SAH}+\mathrm{PBS}$ & $27.78 \%(5 / 18)$ & 0 & Sham & $0 \%(0 / 8)$ & 0 \\
\hline $\mathrm{SAH}+\mathrm{OSM}(100 \mathrm{ng})$ & $33.33 \%(3 / 9)$ & 0 & SAH & $30 \%(3 / 10)$ & 1 \\
\hline $\mathrm{SAH}+\mathrm{OSM}(1 \mu \mathrm{g})$ & $20 \%(2 / 10)$ & 1 & $\mathrm{SAH}+\mathrm{NS}$ & $28.6 \%(4 / 14)$ & 0 \\
\hline $\mathrm{SAH}+\mathrm{OSM}(5 \mu \mathrm{g})$ & $20 \%(2 / 10)$ & 1 & $\mathrm{SAH}+\mathrm{Hep}(5 \mathrm{mg} / \mathrm{kg})$ & $20 \%(2 / 10)$ & 0 \\
\hline \multirow[t]{4}{*}{$\mathrm{SAH}+\mathrm{OSM}(10 \mu \mathrm{g})$} & $10 \%(1 / 10)$ & 1 & $\mathrm{SAH}+\mathrm{Abs}$ & $15.38 \%(2 / 13)$ & 1 \\
\hline & & & $\mathrm{SAH}+\mathrm{Ebs}(4 \mathrm{mg} / \mathrm{kg})$ & $9.1 \%(1 / 11)$ & 0 \\
\hline & & & $\mathrm{SAH}+\mathrm{PBS}$ & $20 \%(2 / 8)$ & 2 \\
\hline & & & $\mathrm{SAH}+\mathrm{OSM}(10 \mu \mathrm{g})$ & $25 \%(2 / 8)$ & 1 \\
\hline Total & & 11 & Total & & 10 \\
\hline
\end{tabular}

other SAH studies. The results indicated that when doses of OSM and ebselen were both introduced at the same time, they had an influence on hepcidin, DMT1, and GPX4 but did not have significant effects on ferroptosis or EBI. This may indeed indicate that hepcidin-induced aggravation of EBI after SAH requires DMT1 involvement. Lastly, heparin is an anticoagulant drug, to avoid its anticoagulant we introduced the glycol-split non-anticoagulant heparin which lost antithrombin-binding affinity, the anticoagulant of glycolsplit non-anticoagulant heparin was studied in HepG2 cells is effective, but whether the anticoagulant of the applied dose of glycol-split non-anticoagulant is effective in SAH, whether it could aggravate bleeding in SAH needs further investigation.

The results of this present study strongly point towards DMT1 as a significant mediator of ferroptosis in the hemorrhagic brain. Both hepcidin and DMT1 appear to be involved in lipid peroxidation and GSH depletion after $\mathrm{SAH}$, which together with a reduced GPX4 activity and increased iron content may lead to EBI via aggravated ferroptosis in several cell compartments.

Concordantly, the suppression of hepcidin and DMT1 alleviates ferroptosis and EBI after SAH. In contrast, the activation of hepcidin aggravates these SAH-induced alter- ations, and this effect is DMT1-dependent. Although further studies are needed, present results provide a novel insight into the disturbed iron homeostasis that may play a central role in mechanisms of EBI after SAH.

\section{Conclusions}

Inhibition of DMT1 by ebselen could suppress iron accumulation and lipid peroxidation, and thereby alleviate ferroptosis and EBI in SAH rats. Heparin downregulated the expression of hepcidin and DMT1, and exerted protective effects that were equivalent to those of ebselen. In addition, OSM increased the expression of hepcidin and DMT1 and aggravated ferroptosis and EBI, while it was reversed by ebselen. Therefore, the study revealed that hepcidin could regulate iron metabolism and contribute to ferroptosis via DMT1 signaling activation in rats with EBI after SAH.

\section{Data Availability}

All the data supporting the results were shown in the paper and can be available from the corresponding authors. 


\section{Conflicts of Interest}

The author(s) declared no potential conflicts of interest with respect to the research, authorship, and/or publication of this article.

\section{Funding}

This study was supported by a grant from the National Natural Science Foundation of China (NSFC 81870927) to Zhaohui He, and a grant from the Natural Science Foundation Project of Chongqing Science and Technology Commission (cstc2019 jcyj-msxmX0239) to Zhaohui He.

\section{Acknowledgments}

Zhaohui He and Hongxia Zhang conceived and designed this research. Hongxia Zhang, Dengzhi Jiang performed this study. Qing Zhao, Xudong Che, Jun Zhao, Xiang Xiang, Yidan Liang, Wang Qin contributed to the data analysis. Hongxia Zhang and Dengzhi Jiang wrote this article. Zhaohui He and Robert Ostrowski reviewed the manuscript for the important intellectual content and helped with writing the final version of the manuscript.

\section{References}

[1] J. Cahill, J. W. Calvert, and J. H. Zhang, "Mechanisms of early brain injury after subarachnoid hemorrhage," Journal of Cerebral Blood Flow and Metabolism, vol. 26, no. 11, pp. 13411353, 2006.

[2] S. Chen, H. Feng, P. Sherchan et al., "Controversies and evolving new mechanisms in subarachnoid hemorrhage," Progress in Neurobiology, vol. 115, pp. 64-91, 2014.

[3] S. J. Dixon, K. M. Lemberg, M. R. Lamprecht et al., "Ferroptosis: An iron-dependent form of nonapoptotic cell death," Cell, vol. 149, no. 5, pp. 1060-1072, 2012.

[4] Y. Li, Y. Liu, P. Wu et al., "Inhibition of ferroptosis alleviates early brain injury after subarachnoid hemorrhage in vitro and in vivo via reduction of lipid peroxidation," Cellular and Molecular Neurobiology, vol. 41, no. 2, pp. 263-278, 2021.

[5] K. Tsuchiya and K. Nitta, "Hepcidin is a potential regulator of iron status in chronic kidney disease," Therapeutic Apheresis and Dialysis, vol. 17, no. 1, pp. 1-8, 2013.

[6] G. Tan, L. Liu, Z. He, J. Sun, W. Xing, and X. Sun, "Role of hepcidin and its downstream proteins in early brain injury after experimental subarachnoid hemorrhage in rats," Molecular and Cellular Biochemistry, vol. 418, no. 1-2, pp. 31-38, 2016.

[7] W. S. Yang and B. R. Stockwell, "Ferroptosis: death by lipid peroxidation," Trends in Cell Biology, vol. 26, no. 3, pp. 165176, 2016.

[8] M. J. Kim, G. J. Yun, and S. E. Kim, "Metabolic regulation of ferroptosis in cancer," Biology, vol. 10, no. 2, p. 83, 2021.

[9] D. Martin-Sanchez, M. Fontecha-Barriuso, J. M. MartinezMoreno et al., "Ferroptosis y nefropatia," Nefrología, vol. 40, no. 4, pp. 384-394, 2020.

[10] D. Tang and G. Kroemer, "Ferroptosis," Current Biology, vol. 30, no. 21, pp. R1292-R1297, 2020.

[11] M. W. Hentze, M. U. Muckenthaler, B. Galy, and C. Camaschella, "Two to tango: regulation of mammalian iron metabolism," Cell, vol. 142, no. 1, pp. 24-38, 2010.
[12] T. Skjorringe, A. Burkhart, K. B. Johnsen, and T. Moos, "Divalent metal transporter $1(\mathrm{dmt} 1)$ in the brain: implications for a role in iron transport at the blood-brain barrier, and neuronal and glial pathology," Frontiers in Molecular Neuroscience, vol. 8, p. 19, 2015.

[13] L. Li, C. Holscher, B. B. Chen, Z. F. Zhang, and Y. Z. Liu, "Hepcidin treatment modulates the expression of divalent metal transporter-1, ceruloplasmin, and ferroportin-1 in the rat cerebral cortex and hippocampus," Biological Trace Element Research, vol. 143, no. 3, pp. 1581-1593, 2011.

[14] X. Zhang, L. X. Li, H. Ding, V. E. Torres, C. Yu, and X. Li, "Ferroptosis promotes cyst growth in autosomal dominant polycystic kidney disease mouse models," Journal of the American Society of Nephrology, vol. 32, no. 11, pp. 27592776, 2021.

[15] X. Zeng, H. An, F. Yu et al., "Benefits of iron chelators in the treatment of Parkinson's disease," Neurochemical Research, vol. 46, no. 5, pp. 1239-1251, 2021.

[16] M. T. Davis and W. J. Bartfay, "Ebselen decreases oxygen free radical production and iron concentrations in the hearts of chronically iron-overloaded mice," Biological Research for Nursing, vol. 6, no. 1, pp. 37-45, 2004.

[17] C. Liu, C. W. Zhang, S. Q. Lo et al., "S-nitrosylation of divalent metal transporter 1 enhances iron uptake to mediate loss of dopaminergic neurons and motoric deficit," The Journal of Neuroscience, vol. 38, no. 39, pp. 8364-8377, 2018.

[18] L. Xie, W. Zheng, N. Xin, J. W. Xie, T. Wang, and Z. Y. Wang, "Ebselen inhibits iron-induced tau phosphorylation by attenuating DMT1 up- regulation and cellular iron uptake," Neurochemistry International, vol. 61, no. 3, pp. 334-340, 2012.

[19] S. Soe-Lin, S. S. Apte, M. R. Mikhael, L. K. Kayembe, G. Nie, and P. Ponka, "Both nrampl and dmt1 are necessary for efficient macrophage iron recycling," Experimental Hematology, vol. 38, no. 8, pp. 609-617, 2010.

[20] M. Poli, D. Girelli, N. Campostrini et al., "Heparin: a potent inhibitor of hepcidin expression in vitro and in vivo," Blood, vol. 117, no. 3, pp. 997-1004, 2011.

[21] M. Poli, M. Asperti, A. Naggi et al., "Glycol-split nonanticoagulant heparins are inhibitors of hepcidin expression in vitro and in vivo," Blood, vol. 123, no. 10, pp. 1564-1573, 2014.

[22] J. Kanda, T. Uchiyama, N. Tomosugi, M. Higuchi, T. Uchiyama, and H. Kawabata, "Oncostatin m and leukemia inhibitory factor increase hepcidin expression in hepatoma cell lines," International Journal of Hematology, vol. 90, no. 5, pp. 545-552, 2009.

[23] B. Chung, F. Verdier, P. Matak, J. C. Deschemin, P. Mayeux, and $\mathrm{S}$. Vaulont, "Oncostatin $\mathrm{m}$ is a potent inducer of hepcidin, the iron regulatory hormone," The FASEB Journal, vol. 24, no. 6, pp. 2093-2103, 2010.

[24] H. Kawabata, T. Uchiyama, S. Sakamoto et al., "A HAMP promoter bioassay system for identifying chemical compounds that modulate hepcidin expression," Experimental Hematology, vol. 43, no. 5, article e405, pp. 404-413.e5, 2015.

[25] J. Y. Lee, O. Sagher, R. Keep, Y. Hua, and G. Xi, “Comparison of experimental rat models of early brain injury after subarachnoid hemorrhage," Neurosurgery, vol. 65, no. 2, pp. 331-343, 2009.

[26] T. Sugawara, R. Ayer, V. Jadhav, and J. H. Zhang, “A new grading system evaluating bleeding scale in filament perforation subarachnoid hemorrhage rat model," Journal of Neuroscience Methods, vol. 167, no. 2, pp. 327-334, 2008. 
[27] Z. He, R. P. Ostrowski, X. Sun et al., "Chop silencing reduces acute brain injury in the rat model of subarachnoid hemorrhage," Stroke, vol. 43, no. 2, pp. 484-490, 2012.

[28] H. Wu, T. Wu, X. Xu, J. Wang, and J. Wang, "Iron toxicity in mice with collagenase-induced intracerebral hemorrhage," Journal of Cerebral Blood Flow and Metabolism, vol. 31, no. 5, pp. 1243-1250, 2011.

[29] M. Fujii, J. Yan, W. B. Rolland, Y. Soejima, B. Caner, and J. H. Zhang, "Early brain injury, an evolving frontier in subarachnoid hemorrhage research," Translational Stroke Research, vol. 4, no. 4, pp. 432-446, 2013.

[30] Y. Xie, W. Hou, X. Song et al., "Ferroptosis: process and function," Cell Death and Differentiation, vol. 23, no. 3, pp. 369379, 2016.

[31] M. Gao, P. Monian, Q. Pan, W. Zhang, J. Xiang, and X. Jiang, "Ferroptosis is an autophagic cell death process," Cell Research, vol. 26, no. 9, pp. 1021-1032, 2016.

[32] B. R. Stockwell, J. P. Friedmann Angeli, H. Bayir et al., "Ferroptosis: a regulated cell death nexus linking metabolism, redox biology, and disease," Cell, vol. 171, no. 2, pp. 273-285, 2017.

[33] J. Y. Cao and S. J. Dixon, "Mechanisms of ferroptosis," Cellular and Molecular Life Sciences, vol. 73, no. 11-12, pp. 2195-2209, 2016.

[34] S. J. Dixon and B. R. Stockwell, "The role of iron and reactive oxygen species in cell death," Nature Chemical Biology, vol. 10, no. 1, pp. 9-17, 2014.

[35] M. Gao, P. Monian, and X. Jiang, "Metabolism and iron signaling in ferroptotic cell death," Oncotarget, vol. 6, no. 34, pp. 35145-35146, 2015.

[36] L. Magtanong and S. J. Dixon, "Ferroptosis and brain injury," Developmental Neuroscience, vol. 40, no. 5-6, pp. 382-395, 2019.

[37] H. Mao, Y. Zhao, H. Li, and L. Lei, "Ferroptosis as an emerging target in inflammatory diseases," Progress in Biophysics and Molecular Biology, vol. 155, pp. 20-28, 2020.

[38] L. Jiang, N. Kon, T. Li et al., "Ferroptosis as a p53-mediated activity during tumour suppression," Nature, vol. 520, no. 7545, pp. 57-62, 2015.

[39] X. Sun, Z. Ou, M. Xie et al., "Hspb1 as a novel regulator of ferroptotic cancer cell death," Oncogene, vol. 34, no. 45, pp. 56175625, 2015.

[40] H. Kuang, T. Wang, L. Liu et al., "Treatment of early brain injury after subarachnoid hemorrhage in the rat model by inhibiting p53-induced ferroptosis," Neuroscience Letters, vol. 762, article 136134, 2021.

[41] J. B. Hansen, M. F. Tonnesen, A. N. Madsen et al., "Divalent Metal Transporter 1 Regulates Iron-Mediated ROS and Pancreatic $\beta$ Cell Fate in Response to Cytokines," Cell Metabolism, vol. 16, no. 4, pp. 449-461, 2012.

[42] L. Viatte, J. C. Lesbordes-Brion, D. Q. Lou et al., "Deregulation of proteins involved in iron metabolism in hepcidin-deficient mice," Blood, vol. 105, no. 12, pp. 4861-4864, 2005.

[43] F. Du, Z. M. Qian, Q. Luo, W. H. Yung, and Y. Ke, "Hepcidin suppresses brain iron accumulation by downregulating iron transport proteins in iron-overloaded rats," Molecular Neurobiology, vol. 52, no. 1, pp. 101-114, 2015.

[44] S. Yamaji, P. Sharp, B. Ramesh, and S. K. Srai, "Inhibition of iron transport across human intestinal epithelial cells by hepcidin," Blood, vol. 104, no. 7, pp. 2178-2180, 2004. 